\title{
Complications and challenges associated with polycystic ovary syndrome: current perspectives
}

\author{
This article was published in the following Dove Press journal: \\ International Journal of Women's Health \\ 31 July 2015 \\ Number of times this article has been viewed
}

\author{
Stefano Palomba' \\ Susanna Santagni' \\ Angela Falbo' \\ Giovanni Battista La Sala ${ }^{1,2}$ \\ 'Unit of Obstetrics and Gynecology, \\ Arcispedale Santa Maria Nuova- \\ Scientific Institute of Treatment \\ and Care (IRCCS), Reggio Emilia, \\ ${ }^{2}$ Department of Obstetrics and \\ Gynecology, University of Modena \\ and Reggio Emilia, Modena, Italy
}

Correspondence: Stefano Palomba Academic Department of Reproductive Medicine and Surgery, ASMN-IRCCS, Viale Risorgimento 80 42123 Reggio Emilia, Italy

Email stefanopalomba@tin.it

\begin{abstract}
Polycystic ovary syndrome (PCOS) represents the most common endocrine dysfunction in fertile women and it is considered a heterogeneous and multifaceted disorder, with multiple reproductive and metabolic phenotypes which differently affect the early- and long-term syndrome's risks. Women with PCOS present an adverse reproductive profile, including a high risk of pregnancy-induced hypertension, preeclampsia, and gestational diabetes mellitus. Patients with PCOS present not only a higher prevalence of classic cardiovascular risk factors, such as hypertension, dyslipidemia, and type-2 diabetes mellitus, but also of nonclassic cardiovascular risk factors, including mood disorders, such as depression and anxiety. Moreover, at the moment, clinical data on cardiovascular morbidity and mortality in women with PCOS are controversial. Finally, women with PCOS show an increased risk of endometrial cancer compared to non-PCOS healthy women, particularly during premenopausal period. Currently, we are unable to clarify if the increased PCOS early- and long-term risks are totally due to PCOS per se or mostly due to obesity, in particular visceral obesity, that characterized the majority of PCOS patients. In any case, the main endocrine and gynecological scientific societies agree to consider women with PCOS at increased risk of obstetric, cardiometabolic, oncology, and psychological complications throughout life, and it is recommended that these women be accurately assessed with periodic follow-up.
\end{abstract}

Keywords: cardiovascular disease, infertility, polycystic ovary syndrome, PCOS, pregnancy

\section{Introduction}

Polycystic ovary syndrome (PCOS) is a common endocrine disease in women, characterized by heterogeneous presentation of hyperandrogenism, ovulatory dysfunction, and polycystic ovarian morphology (PCOM). Despite PCOS being considered the most common female endocrinopathy during the reproductive life, ${ }^{1,2}$ the prevalence estimate greatly varies, ranging from $6 \%$ to $10 \%{ }^{1,3,4}$ depending on the diagnostic criteria used $^{5}$ and on the multiple faces with which this complex syndrome occurs.

The first recognition of the disease goes back to 1935 with Stein and Leventhal description, ${ }^{6}$ since then many scientific societies and workshop groups developed different diagnostic criteria, with the aim to provide a more inclusive definition of the syndrome. The National Institute Health $(\mathrm{NIH})$ diagnostic criteria were based on the results of a survey among experts who considered a woman with PCOS if she presented with the combination of chronic oligo- or anovulation and clinical or biochemical signs of hyperandrogenism, with the exclusion of other related endocrine disorders. ${ }^{7}$ In 2003, the European Society of Human Reproduction and Embryology (ESHRE)/American Society of Reproductive Medicine (ASRM)-Sponsored PCOS Consensus Workshop Group suggested, after an international meeting held in Rotterdam, the addition of a third criteria, ie, the presence of PCOM, establishing the 
PCOS diagnosis by the presence of at least two of these three criteria (chronic anovulation, hyperandrogenism, and PCOM on ultrasonography). ${ }^{8}$ Over the years, in the light of the ongoing clinical and metabolic relevance of the hyperandrogenism, ${ }^{9-11}$ the Androgen Excess and PCOS (AEPCOS) Society postulated the androgen excess as a central feature of the disease and PCOS should be defined by the presence of hyperandrogenism (clinical and/or biochemical) in combination with ovarian dysfunction (oligoanovulation and/or PCOM), with the exclusion of related disorders from other causes. ${ }^{12}$ That criteria were based on the best scientific evidences available on the issue. In 2011, the Amsterdam ESHRE/ASRM-Sponsored Third PCOS Consensus Workshop Group ${ }^{13}$ identified different phenotypes, according to the different criteria's combinations and separated the most classic phenotype, characterized by hyperandrogenism and chronic anovulation, from those characterized by ovarian dysfunction and PCOM. On December 2012, the NIH Evidence-Based Methodology Workshop on PCOS ${ }^{14}$ confirmed and recommended to maintain the broad diagnostic Rotterdam criteria along with the identification of the specific PCOS phenotypes for each single patient, especially for their different potential cardiometabolic implications; indeed the identification of specific phenotypes in women with PCOS seems to be justified from the metabolic point of view. ${ }^{15}$ More recently, in the Practice Guidelines of the Endocrine Society, the use of the Rotterdam criteria for PCOS diagnosis was confirmed, ${ }^{16}$ even if the characterization of the specific phenotype at diagnosis was not considered clinically needed. This crucial point is still under debate in the scientific community. ${ }^{17}$

Several articles have been published on the short- and long-term effects of PCOS on the women's health due to an increased incidence of early complications such as a worsening of fertility and obstetric outcomes and to an increased rate of late complications as well as enhanced cardiovascular, metabolic, and oncology risks. It is very difficult to accurately define the precise extent of these complications, due to the heterogeneous nature of the syndrome, the unclear pathogenetic mechanisms, and the presence of confounding factors, such as obesity. Moreover, the PCOS phenotypes in women change across the life span; ${ }^{18}$ therefore, the changes in ovarian function and in the metabolic regulation could modify the disease's expression and that may play a role in the morbidity of the syndrome during the late reproductive age and menopause.

In line with these considerations, the current review was aimed at summarizing the current knowledge and perspectives about the short- and long-term complications of PCOS on the women's health and longevity, considering PCOS not only as a reproductive age disorder but as a longlife syndrome.

\section{Early-term complication Infertility}

Infertility was one of the main symptoms originally attributed to the PCOS according to the first description. ${ }^{6}$ Subsequent epidemiologic evidences suggested that PCOS is the most common cause of ovulatory disorder and oligoanovulation is related with increased risk for infertility. ${ }^{19}$ In a large population of 1,741 women affected by PCOS, primary infertility was reported in $50 \%$ of women, while secondary infertility was reported in $25 \%$ of women. ${ }^{20}$

Several PCOS comorbidities seemed to contribute to infertility. In particular, insulin resistance (IR $)^{21}$ and obesity ${ }^{22}$ were independently related to an increased risk of abortion and to reduced pregnancy and live-birth rates. Endometrial abnormalities were also reported in PCOS women, ${ }^{23,24}$ affecting potentially the implantation. Finally, ovarian alterations at several levels were described, ie, ovarian/follicular/corpus luteum vascularity, ${ }^{25}$ follicular fluid environment, ${ }^{26,27}$ and subsequent oocytes competence ${ }^{26}$ and quality. ${ }^{28}$

Notwithstanding that theoretical reproductive abnormalities related to the syndrome, the available studies based on modern PCOS diagnostic criteria and with sample sizes sufficiently large seemed to report conflicting results. ${ }^{29-32}$

The typical PCOM appeared to diminish with increasing age. ${ }^{33}$ Similarly, menstrual cycles become normalized with increasing age in PCOS women. ${ }^{29,34}$ Finally, a recent study demonstrated that the reproductive outcome of women with a previous diagnosis of PCOS was similar to that of a nonPCOS population. ${ }^{32}$ In particular, ovarian reserve seemed to be better preserved in PCOS women, ${ }^{32}$ resulting in a larger duration of the reproductive window during aging. ${ }^{35}$ The livebirth rate and the rate of miscarriages were similar in PCOS patients and control women, and in more than two-thirds of the PCOS women, pregnancy occurred spontaneously. ${ }^{32}$ Similar pregnancy and live-birth rates between PCOS and control women were also reported after conventional in vitro fertilization (IVF) by a meta-analysis. ${ }^{36}$

International documents ${ }^{13}$ highlighted the lack of clear data on the risk of miscarriage in women with PCOS, and that PCOS should be not considered per se a risk factor for miscarriage. The most recent guidelines of the Endocrine Society-appointed Task Force of Experts ${ }^{16}$ suggested that PCOS is a risk factor for infertility only in the presence of 
oligoanovulation; thus they recommended screening ovulatory status using menstrual history in all women with PCOS seeking fertility.

\section{Obstetric complications}

PCOS has historically been defined as a syndrome related to ovulatory infertility. Today, especially with the introduction of the new diagnostic criteria, ${ }^{37}$ the focus has shifted to reproductive problems, including also the obstetrics complications. Moreover, the obstetric risk may be exacerbated by comorbidities, such as obesity and/or IR typical of the syndrome.

From a pathogenetic point of view, the increased incidence of pregnancy complications in women with PCOS can be the result of several factors, such as PCOS features, infertility treatments, multiple pregnancies, obesity, IR and metabolic dysfunction, inflammation, and placental alterations. ${ }^{38}$ In practice, several data uncontrolled for body mass index (BMI), including mostly retrospective or prospective studies with relatively small samples, have been published on maternal, neonatal, and obstetric complications in women with PCOS. ${ }^{38}$ These data were described and analyzed in three systematic reviews with meta-analysis. ${ }^{39-41}$

It is still debated whether women with PCOS have an increased risk of miscarriage compared to women without PCOS. In the PCOS consensus 2012, miscarriage rates are suggested to be comparable between women with and without PCOS, although data show conflicting results. ${ }^{13}$ A meta-analysis of studies concerning women with and without PCOS undergoing IVF demonstrated no difference in miscarriage rates. ${ }^{36}$ This result was confirmed in a recent large cohort study. ${ }^{42}$ Moreover, most women with PCOS become pregnant by using ovulation induction medications that can modify the risk of miscarriage compared to women with natural conception. ${ }^{38}$

All meta-analyses on pregnancy complications ${ }^{39-41}$ were in agreement in reporting an increased risk of pregnancyinduced hypertension or preeclampsia in women with PCOS of at least threefold. That data, obtained from retrospective studies, have been recently confirmed by a prospective study. ${ }^{43}$ Specifically, the risk of pregnancy-induced hypertension and preeclampsia resulted of $12.7 \%$ and $8 \%$, respectively, and significantly higher than those observed in healthy controls (5.3\% and $2 \%$, respectively).

The gestational diabetes mellitus (GDM) is the most commonly described pregnancy complication in women with PCOS with a threefold risk and an absolute risk of $6 \%-15 \% .{ }^{39-41}$ Two recent prospective studies confirmed an increased incidence of GDM of up to $14.7 \% 0^{43,44}$ and $22 \%$ in women with PCOS. ${ }^{45}$

Data on the risk of cesarean section, as well as those on the risk for adverse fetal outcomes, in women with PCOS are controversial, ${ }^{39-41}$ whereas no significant effect of PCOS on the risk for operative vaginal delivery has been detected. ${ }^{39,40}$ Recently, the risk of preterm delivery was increased twofold and more in PCOS patients, even if confined to hyperandrogenic subjects. ${ }^{46}$ Neonates born to women with PCOS had a twofold increased risk for admission to the neonatal intensive care unit ${ }^{41}$ and their mortality was increased of threefold. ${ }^{39}$ Albeit the first published meta-analysis ${ }^{39}$ found no difference in risk of small-for-gestational age neonates, the most recent one found an almost twofold increased risk of small-for-gestational age and no risk of large-for-gestational age neonates. ${ }^{40}$ Two recent studies confirmed an increased risk of small-for-gestational age of four- ${ }^{47}$ and twofold and half $^{43}$ in neonates of women with PCOS, whereas another study showed no effect of PCOS on the risk of small-forgestational age. ${ }^{46}$ On the other hand, an increased incidence of large-for-gestational age in PCOS patients was observed in a retrospective ${ }^{48}$ and in a prospective study. ${ }^{43}$ The incidence of macrosomia, however, was similar in PCOS women when compared to controls. ${ }^{39}$

On the basis of these considerations, recently, the recent Endocrine Society guidelines for the diagnosis and treatment of PCOS ${ }^{16}$ declared that women with PCOS are at increased risk of pregnancy complications recommending preconceptual assessment of BMI, blood pressure, and oral glucose tolerance. ${ }^{16}$

\section{Long-term complications Cardiovascular risk}

As reported by the main scientific societies, ${ }^{13,15,49}$ women with PCOS present an increased prevalence of classic risk factors for cardiovascular disease (CVD) such as hypertension, dyslipidemia, diabetes, and obesity and nonclassic risk factors such as C-reactive protein (CRP), homocysteine, and tumor necrosis factor- $\alpha .^{50}$ PCOS at any age is characterized by greater odds for elevated CVD risk markers and these elevated makers can occur without obesity but are magnified with obesity. ${ }^{13}$

In 2004, a worldwide case-control study of patients from 52 countries was published, the INTERHEART study, ${ }^{51}$ that found nine potentially modifiable risk factors, accounted for over $94 \%$ of the population-attributable risk of a first myocardial infarction in women; the nine factors included smoking, hypertension, dyslipidemia, diabetes, visceral 
obesity, psychosocial factors, decreased consumption of fruits and vegetables, regular consumption of alcohol, and regular physical activity. The majority of these occur in the PCOS woman. It is estimated that the prevalence of each risk factor is approximately double for women with PCOS when compared with controls, while it is 1.5 times higher in BMI-matched studies beginning in adolescence and it is found in every decade. ${ }^{13}$

A study conducted in Brazil, ${ }^{52}$ regarding prevalence of hypertension in women with and without PCOS, revealed a twofold prevalence of the disorder in women with PCOS. The increased risk of hypertensive state seems to be explained by IR and hyperinsulinemia, typical of PCOS, that alter vascular smooth muscle cells causing hypertrophy of vascular muscle wall with reduced compliance and by the interference in the endothelium-dependent vasodilatation mechanisms. ${ }^{53}$ Also high testosterone levels, but not sex hormone-binding globulin (SHBG) levels, increased the hypertension's risk, even when adjusted for age, BMI, and other anthropometric and hormonal parameters. ${ }^{54} \mathrm{~A}$ Swedish study ${ }^{55}$ also showed that, even in the absence of a real hypertensive state, PCOS women presented a significantly higher daytime systolic blood pressure, mean arterial values of blood pressure, and an increased pulse rate, than healthy controls. This significant prehypertensive state remained even after adjusting for BMI, body fat distribution, and IR. ${ }^{55}$

Dyslipidemia is very common in PCOS patients, ${ }^{56}$ which is present in $70 \%$ of patients in the United States (US) with different patterns. ${ }^{57}$ Most often it is represented by hypertriglyceridemia and low high-density lipoprotein (HDL) cholesterol levels and small dense low-density lipoprotein (LDL) cholesterol particles (also called atherogenic lipoprotein phenotype), ${ }^{58,59}$ similar to that found in type- 2 diabetes mellitus (T2DM) and typical for the states of IR, ${ }^{60}$ while the increased LDL cholesterol in PCOS is less dependent on body weight and may be partially related to the hyperandrogenism. ${ }^{61}$ Higher non-HDL cholesterol, frequent in women with PCOS, reflects altered ApoB/A1 ratios, an important risk factor for CVD. ${ }^{61,62}$ Moreover, altered ApoB/ApoA ratios reflect also a more atherogenic lipoprotein lipid pattern in women with PCOS. The current evidences show that different lipid patterns may be present in women with PCOS $^{49}$ but the impact of dyslipidemia in different PCOS phenotypes is not known. Dyslipidemia may deteriorate with obesity but the influence of BMI on the severity of dyslipidemia is still controversial $^{61}$ and may be influenced also by the origin of investigated PCOS women. ${ }^{63}$ In fact, differences between ethnic and geographical background are likely to depend on the combination of genetic, environmental, and hormonal factors.

Among the cardiovascular risk factors, T2DM represents one of the most important and PCOS is considered a major risk factor for developing impaired glucose tolerance (IGT) and T2DM, with a level A of evidence according to ESHRE/ASRM statement of the Third PCOS Consensus Workshop Group. ${ }^{13}$ PCOS is an independent risk factor for the development of T2DM and the progression of IR to glucose intolerance and finally T2DM is variably. However, it is estimated that, in a third of those affected, T2DM occurs within 2-3 years ${ }^{64}$ and exceed $50 \%$ within 10 years. ${ }^{65}$ The negative association between glucose metabolism and PCOS is worsened by the presence of obesity; there is approximately three to four times more likeliness to develop diabetes than in women without PCOS or obesity but the association remains even in women with a BMI of less than $25 \mathrm{~kg} / \mathrm{m}^{2}$ and in a BMI-matched study. ${ }^{66,10}$

More and more scientific evidences suggest a role of nonclassic CVD risk factors, related to a systemic inflammatory state, in PCOS patients, such as CRP. CRP is commonly considered a vascular inflammatory marker that predicts the

Table I Summary of recommendations for the management of infertility in oligoanovulatory women with PCOS

\begin{tabular}{ll}
\hline Management of early complications & First-line nonpharmacologic approach to treat obese/overweight \\
Lifestyle modification & oligoanovulatory PCOS patients \\
(diet and physical activity) & First-line treatment of ovulatory infertility in women with PCOS \\
Clomiphene citrate & $\begin{array}{l}\text { Potential first-line treatment of ovulatory infertility but it is still off-label drug } \\
\text { not recommended in clinical practice }\end{array}$ \\
Letrozole & Treatment of choice as clomiphene citrate sensitizing and as adjuvant therapy to \\
Metformin & prevent OHSS in PCOS women undergoing IVF cycle, but it is still off-label drug \\
& Indicated in well-selected cases of oligoanovulatory PCOS \\
Laparoscopic ovarian drilling & women who need laparoscopic assessment of the pelvis \\
& Considered last treatment option in PCOS population \\
Gonadotropins & for high costs, high risk of multiple pregnancies, and OHSS \\
\hline
\end{tabular}

Abbreviations: IVF, in vitro fertilization; OHSS, ovarian hyperstimulation syndrome; PCOS, polycystic ovary syndrome. 
Table 2 Summary of recommendations for clinical assessment and treatment of long-term PCOS complications

\begin{tabular}{|c|c|c|}
\hline \multicolumn{3}{|c|}{ Management of long-term complications } \\
\hline & Clinical assessment & Therapeutic approaches \\
\hline Metabolic risk & $\begin{array}{l}\text { Screening for impaired glucose tolerance and T2DM } \\
\text { with } 75 \text { g OGTT in PCOS women with: } \\
\text { - age }>40 \text { years } \\
\text { - BMI }>30 \\
\text { - classic phenotype } \\
\text { - presence of achantosis nigricans } \\
\text { - personal and/or family history of T2DM } \\
\text { BMl and waist circumference at every visit: } \\
\text { - waist circumference }>80 \mathrm{~cm} \\
\text { - abdominal obesity } \\
\text { Periodic reassessment with OGTT }\end{array}$ & $\begin{array}{l}\text { Lifestyle change programs (hypocaloric diet and } \\
\text { physical exercise) represent the first-line approach } \\
\text { for obese PCOS women } \\
\text { Metformin use for prevention of diabetes in PCOS } \\
\text { women with impaired glucose tolerance when lifestyle } \\
\text { modification is not successful and/or as an adjuvant to } \\
\text { general lifestyle modifications } \\
\text { Thiazolidinediones as alternative therapy in insulin- } \\
\text { resistant, obese PCOS patients who are intolerant } \\
\text { or refractory to metformin, or with severe insulin } \\
\text { resistance due to genetic disorder }\end{array}$ \\
\hline Cardiovascular risk & $\begin{array}{l}\text { CVD risk assessment at any age with: } \\
\text { - blood pressure } \\
\text { - lipid profle } \\
\text { - waist circumference } \\
\text { - BMI } \\
\text { - glucose profile } \\
\text { - cigarette smoking } \\
\text { - family history of early CVD } \\
\text { - evaluation for depression, anxiety, and quality-of-life } \\
\text { Categorize PCOS patients as "at risk" } \\
\text { for CVD if present: } \\
\text { - obesity } \\
\text { - hypertension } \\
\text { - dyslipidemia } \\
\text { - cigarette smoking } \\
\text { - subclinical vascular disease } \\
\text { - impaired glucose tolerance } \\
\text { - family history of premature CVD } \\
\text { Categorize PCOS patients as "at high risk" } \\
\text { for CVD if present: } \\
\text { - metabolic syndrome } \\
\text { - T2DM } \\
\text { - vascular and/or renal disease } \\
\text { Periodic clinical reassessment }\end{array}$ & $\begin{array}{l}\text { Lifestyle modification: } \\
\text { - diet } \\
\text { - physical exercise } \\
\text { - smoking cessation } \\
\text { Metformin use for prevention of T2DM in PCOS } \\
\text { women with impaired glucose tolerance when lifestyle } \\
\text { modification is not successful and/or as an adjuvant to } \\
\text { general lifestyle modifications } \\
\text { Statins to lower LDL-C levels } \\
\text { Antihypertensive drugs }\end{array}$ \\
\hline Oncological risk & $\begin{array}{l}\text { In presence of amenorrhoic patients or abnormal uterine } \\
\text { bleeding, assessment for the presence of endometrial cancer with } \\
\text { ultrasound and/or endometrial biopsy }\end{array}$ & $\begin{array}{l}\text { Periodic progestogen withdrawal (at least four } \\
\text { episodes per year) should be indicated in anovulatory } \\
\text { PCOS women }\end{array}$ \\
\hline
\end{tabular}

Abbreviations: CVD, cardiovascular disease; OGTT, oral glucose tolerance test; PCOS, polycystic ovary syndrome; LDL-C, low-density lipoprotein cholesterol; BMI, body mass index; T2DM, type-2 diabetes mellitus.

development of $\mathrm{CVD}^{67}$ and T2DM. ${ }^{68}$ Studies reported higher CRP levels in women with PCOS, ${ }^{69}$ although the serum CRP levels seem to be more associated with obesity than with the presence of PCOS per se. ${ }^{70}$

Plasma homocysteine levels are widely accepted as an independent CVD risk factor and many different scientific papers have shown elevated plasma homocysteine levels in PCOS women, ${ }^{71,72}$ also independent of BMI, ${ }^{73}$ as a consequence of the negative effects of IR and hyperinsulinemia on the homocysteine metabolism.

Other many biochemical inflammatory and thrombotic markers of cardiovascular risk have been reported in excess in women with PCOS compared with non-PCOS controls, ${ }^{74,75}$ such as tumor necrosis factor- $\alpha$, interleukin-6 (IL-6), IL-18, IL-17, factor VIIc, tissue plasminogen activator (t-PA), fibrinogen, von Willebrand factor (vWF), plasminogen activator inhibitor-1 (PAI-1), thrombo-modulin, D-dimers, antithrombin III (ATIII), Sp-Selectin, endothelin-1 (ET-1), asymmetric dimethylarginine (ADMA), intercellular adhesion molecule-1 (ICAM-1), soluble vascular cell adhesion molecule-1 (sVCAM-1), serum advanced glycation end-products (AGEs), membrane receptor for AGEs (RAGE), malondialdehyde (MDA), nitric oxide (NO), and latency-associated peptides (LAPs). ${ }^{13}$ 
Nevertheless, despite the higher prevalence of CVD risk markers in PCOS women than in healthy controls, the association of these markers with cardiovascular events remains unclear. Women with PCOS have more subclinical vascular disease than healthy women but evidence for increased CVD morbidity and mortality in these women, based upon Rotterdam and/or NIH criteria, remains inconclusive..$^{30,76,77}$

Retrospective studies show that typical features of PCOS, such as increased waist/hip ratio, hirsutism, or PCOM, are more commonly related to coronary artery disease in women undergoing coronary angiography. ${ }^{78,79}$ In asymptomatic women with PCOS, the presence of subclinical atherosclerosis has been determined using different invasive and more frequently noninvasive methods, as well as structural markers: endothelial function, carotid intima media thickness (CIMT), and coronary artery calcification (CAC) score. Endothelial dysfunction, early sign of atherosclerosis, can be assessed by examining artery's flow-mediated dilatation ${ }^{80}$ and, in a recent meta-analysis, ${ }^{81}$ this parameter, measured at brachial artery, was found to be lower in women with PCOS compared to controls, even after controlling for age, BMI, and smoking. Endothelial dysfunction was shown to be associated with higher levels of androgens and with IR, ${ }^{82}$ also observed at very early ages, with a trend of worsening from lean to overweight and obese PCOS women. ${ }^{83}$ Another assessment parameter of subclinical atherosclerosis is measurement of CIMT. Increased CIMT correlates with a rise of CVD risk and a recent systematic review ${ }^{84}$ confirmed a thicker CIMT in women with PCOS compared to non-PCOS controls. Finally, CAC is a valid coronary atherosclerosis marker, used to assess the risk of myocardial infarction or sudden cardiac death; $; 5$ a recent systematic review ${ }^{86}$ of the studies showed that PCOS women have also been found to present a greater prevalence of CAC than unaffected women, and this was independent of age and BMI. These markers of subclinical atherosclerosis are correlated with age, components of metabolic syndrome, IR, and circulating androgen levels. ${ }^{87-89}$

Therefore, it could be assumed that the lifelong metabolic dysfunctions of PCOS are responsible for a worse cardiovascular profile and predispose for CVD with aging. Indeed, few studies aimed to investigate the true occurrence of CVD in PCOS women and most of them include women with a retrospective diagnosis of PCOS and/or studies not based on currently diagnostic accepted criteria. A 21-year controlled follow-up study, ${ }^{90}$ in a Swedish cohort of PCOS women, did not find an increased rate of myocardial infarction, stroke, or mortality, despite the higher prevalence of hypertension and dyslipidemia in that population. On the contrary, a recent meta-analysis ${ }^{91}$ found an increased incidence of cardiovascular events in women with PCOS, but did not distinguish between coronary heart disease and stroke. Finally, a more recent systematic review and meta-analysis ${ }^{92}$ showed that women with PCOS appear to be at increased risk of nonfatal stroke and possibly coronary heart disease, although the data synthesis did not provide evidence of increased risk in CVD in PCOS entirely independent of BMI.

To date, uncertainty exists to whether PCOS per se increases cardiovascular mortality and the impact of various lifestyle factors and of the different PCOS treatments, such as oral contraceptive pill, anti-androgens, insulin sensitizers, or laparoscopy ovarian diathermy on cardiovascular risk of these patients. A registry for patients with CVDs and a multisite longitudinal follow-up study or case-control study is urgently needed. ${ }^{13}$

\section{Metabolic risk}

Actually, obesity is considered a serious growing epidemic disorder in the world population, especially in the childhood and teenage years. Women with PCOS represent a cohort of people with a high prevalence of overweight (BMI $>25$ $\mathrm{kg} / \mathrm{m}^{2}$ ) and obesity (BMI $>30 \mathrm{~kg} / \mathrm{m}^{2}$ ) compared to healthy women, up to $61 \%$ of prevalence, ${ }^{93}$ with a typical central distribution of adipose tissue, although there is a wide variability in the estimates of obesity in PCOS women across different countries and ethnicities. ${ }^{13}$ In the Western countries, such as Australia, USA, and UK, the PCOS women have the highest prevalence of overweight and obesity, ${ }^{94,95}$ compared to Chinese PCOS women (only $20 \%$ has a BMI of $25 \mathrm{~kg} / \mathrm{m}^{2}$ or greater). ${ }^{96}$ The causal role in the association between obesity and PCOS has yet to be determined but cultural, lifestyle, and ethnic factors take part in these conditions. Recent literature have found that greater adiposity was described in more severe reproductive phenotypes (characterized by hyperandrogenism associated with chronic anovulation), ${ }^{49}$ and lean women had milder reproductive phenotype compared with those who were overweight; ${ }^{97}$ so obesity can exacerbate the PCOS reproductive phenotypes. It also appears that obesity could have a bidirectional relationship with PCOS, as women with PCOS are more inclined to weight gain and excessive weight gain increases PCOS prevalence, unmasking a latent PCOS condition, but this yet remains to be clarified..$^{98}$

Women with a diagnosis of PCOS are more likely to have upper body fat distribution, even in the absence of an obesity condition and independent of BMI levels. The 
android fat distribution represents the most metabolic relevant adipose tissue. In fact, the greatest health implications of PCOS are associated with excess weight and abdominal circumference ${ }^{99}$ because a greater visceral adiposity is associated with a greater IR, ${ }^{100,101}$ which is considered one of the most important metabolic pathogenetic key of the syndrome. Visceral obesity correlates with greater fasting insulin levels and greater insulin area under the curve. ${ }^{100,101}$ Moreover, excess central adiposity reflects a worsened dyslipidemic profile, with higher triglyceride levels and low HDL cholesterol levels. ${ }^{102,103}$

IR, an essential etiological factor of PCOS, was traditionally attributed primarily to obesity. Moreover, it showed the presence of an intrinsic IR in PCOS, independent of obesity, supported by some evidences, such as insulin-signaling abnormalities due to excessive serine phosphorylation on insulin receptor and to defects in post-receptor signaling molecules. ${ }^{104,105}$ The cellular and molecular mechanisms of IR in PCOS differ from those of IR that occurs in other metabolic disease, such as T2DM and obesity. However, the synergistic negative effect of PCOS and obesity on insulin action is well-recognized, as hepatic IR is present only in obese women with PCOS. ${ }^{105}$ Nevertheless, IR occurs also without obesity and its prevalence in lean women with PCOS is approximately of $75 \%{ }^{97}$

IR and associated hyperinsulinemia act by increasing androgen production by ovaries and decreasing hepatic SHBG production, resulting in free androgens excess. ${ }^{106}$ The amount of androgen excess worsens with obesity even if the association between body fat and hyperandrogenism seems to be indirect and, at least in part, mediated by IR and the associated hyperinsulinemia. ${ }^{107}$

Obesity and overweight increase the risk of developing T2DM in PCOS women, although, as previously reported, the PCOS represents an independent risk factor for T2DM. ${ }^{108}$ In particular, the syndrome is considered a significant risk factor for development of DM both in later life and in young overweight or obese women with PCOS. ${ }^{13}$ In a recent systematic review and meta-analysis, the T2DM risk was increased fourfold compared with BMI-matched control groups while the odds ratio (OR) for IGT was 2.5. ${ }^{10}$ The likelihood of developing T2DM significantly increased as BMI, fasting glucose, and glucose area under the curve (AUC) at baseline increased and significantly decreased as SHBG levels at follow-up increased. ${ }^{109}$

The cardiometabolic profile of women with PCOS seems to depend on the different PCOS reproductive phenotypes, where women with hyperandrogenic PCOS (classic
NIH-criteria PCOS) have a worse cardiometabolic profile and higher prevalence of CVD risk factors compared with women with non-hyperandrogenic PCOS. ${ }^{13,110}$ In particular, in women with hyperandrogenic PCOS, the prevalence of metabolic syndrome has been recently estimated at approximately $25.8 \%,{ }^{110}$ in line with other prevalence's valuations according to the former $1992 \mathrm{NIH}$ criteria. ${ }^{10}$ This rate of metabolic syndrome is significantly increased compared with that in reproductively healthy women of similar age and weight ${ }^{9,111}$ and compared with that in non-hyperandrogenic women with PCOS. ${ }^{110}$ This increased risk of metabolic syndrome may be explained by a severe IR associated with classic NIH-criteria PCOS and by hyperandrogenism, possible independent contributor to metabolic syndrome risk. ${ }^{105}$ Moreover, women with PCOS, diagnosed according to the Rotterdam criteria (including other phenotypes than hyperandrogenism and chronic anovulation), have much milder metabolic dysfunction or are even metabolically normal. ${ }^{112,113}$ However, it was also found that similar cardiometabolic profiles are present where the different phenotypes present similar BMI or similar amount of visceral obesity or where the differences are corrected for adiposity; so it has been suggested that metabolic phenotype parallels reproductive phenotype but obesity can exacerbate the differences among the metabolic phenotypes. ${ }^{114}$

With regards of IGT and T2DM risk across the PCOS phenotype, a systematic review did not report differences in T2DM prevalence between the milder reproductive phenotypes and the more severe phenotypes ${ }^{113}$ and this issue has been later confirmed in a cross-sectional study where women with NIH and non-NIH PCOS have similar elevated T2DM risk, relative to controls, independent of age and adiposity, requiring a similar clinical screening and treatment for T2DM for both phenotypes of PCOS. ${ }^{115}$

\section{Oncology risk}

Since PCOS is considered as a lifelong multisystemic and multifaceted disorder, the reproductive and metabolic alterations characterizing the syndrome may be also associated with an increased risk of the development of cancers, such as the endometrial, ovarian, and breast cancer, which recognize potential hormonal and/or metabolic pathogenetic mechanisms.

The first papers reporting an association between PCOS and endometrial cancer date back to the forties and fifties, but, to date, the lack of exhaustive evidence of the relationship between PCOS and gynecological malignancies can be mainly explained by the relative lack of studies compared with those 
investigating cardiovascular morbidity in PCOS women ${ }^{116}$ and by the small number of cases in each study. The potential mechanisms which could promote the onset of neoplastic diseases in these women, particularly endometrial cancer, include the chronic anovulatory state, resulting in an unopposed estrogen action, associated with hyperandrogenism. ${ }^{117}$

At present, based on the most recent meta-analysis, ${ }^{118}$ women with PCOS of all ages seem to be at an increased risk of endometrial cancer. In particular, the risk of endometrial cancer may be even higher in the premenopausal subgroup of women with PCOS, while overall the risk of ovarian and breast cancer was not significantly increased. ${ }^{118}$ These current results are consistent with previous reviews published on cancer in $\operatorname{PCOS}^{116,119,120}$ but in contrast with a previous systematic review ${ }^{121}$ which found that women with PCOS were also at increased risk of ovarian cancer. That review, however, was lacking in meta-analytic analysis and included only one study about ovarian cancer in PCOS.

Unfortunately, as with studies of cardiovascular risk, the assessment of cancer risk in PCOS women is complicated by the presence of various potential confounding factors such as obesity, T2DM, inflammation, and metabolic syndrome, which are highly represented in PCOS populations. Obesity is a recognized risk factor for endometrial cancer and the authors of the most recent meta-analysis ${ }^{118}$ acknowledge that the increased risk of this cancer could be attributed, at least in part, to increased prevalence of obesity in PCOS women. The same issue applies to T2DM, another possible confounding factor that is most representative in PCOS women and associated with an higher risk of endometrial cancer, possibly secondary to hyperinsulinemia, hyperglycemia, and inflammation. ${ }^{122}$ Therefore, there is uncertainty as to whether increased endometrial cancer risk is due to different metabolic risk factors or PCOS itself, which is characterized by many metabolic and reproductive complications that could be responsible for an increased oncology risk on endometrium. Moreover, it is difficult to estimate the strength of this association because the PCOS diagnoses used in many of the studies are based on self-reports ${ }^{123,124}$ or on unusual criteria, ${ }^{125}$ and, mostly, the definition of PCOS has changed over time with limitations also in control groups.

Nonetheless, it is generally accepted that PCOS women with amenorrhea are at greater risk for endometrial hyperplasia and cancer, ${ }^{13}$ therefore, ESHRE/ASRM Consensus Workshop Group has established a proper endometrial surveillance with ultrasound and/or biopsy to assess endometrial thickening in women which experience extended period of amenorrhea, based on clinical suspicion and presentation, and in these women periodic progestogen withdrawal is also recommended, at least four episodes per year. ${ }^{13}$

Agents that induce ovulation and improve the chance of pregnancy would also combat the unopposed estrogen and possibly lower the risk of endometrial hyperplasia and cancer. ${ }^{99}$ In the same way, metformin, an insulin-sensitizing agent, has been shown to exert a chemoprotective and antiproliferative effect on numerous cancers, ${ }^{126,127}$ and it has been suggested to have an antitumor effect on endometrial cancer. ${ }^{128}$ To date, however, there are scarce research data to support metformin use in PCOS women for endometrial cancer prevention in the clinical practice. ${ }^{129,130}$

There is limited and contradictory evidence regarding the risk of ovarian ${ }^{131}$ and breast cancer ${ }^{121}$ in women with PCOS; so currently, the ESHRE/ASRM consensus statement ${ }^{13}$ does not recommend routine surveillance strategy and/or clinical care to detect ovarian and breast cancer in women with PCOS. There is also insufficient evidence to evaluate any association of PCOS with vaginal, vulvar, and cervical cancer.

\section{Other disorders}

In addition to well-known cardiovascular and metabolic impairments, patients with PCOS present an increased risk for psychological disorders and reduced quality-of-life (QoL) compared to healthy women. ${ }^{132-135}$ In a recent meta-analysis, it has been found that the prevalence rates of depression in PCOS range from $14 \%$ to $67 \%$, with a fourfold greater odds of depressive symptoms compared with age-matched control women. ${ }^{132}$ Moreover, this greater risk is sustained also after subanalysis of BMI-matched subjects. ${ }^{132} \mathrm{~A}$ following systematic review of the literature showed an increased prevalence of generalized anxiety and an increase in mean anxiety scores in women with PCOS compared with control women. ${ }^{136}$ Limited data are published about anxiety score in adolescents with PCOS, revealing a slight increase in anxiety in PCOS young women. ${ }^{137}$

The assessment of psychological symptoms and QoL can be performed with different validated disease-specific questionnaires or structured interviews, such as the Hospital Anxiety and Depression Scale, the Beck Anxiety Inventory, and the Beck Depression Inventory, that evaluate the frequency of mental symptoms in anxiety and depression, respectively. A further questionnaire that addresses many areas including depression and anxiety is the Symptom Checklist 90. The Health-Related QoL has been investigated by generic questionnaires, such as the Short Form-36 (SF-36) questionnaire, the most frequently utilized instrument for many diseases besides PCOS, or by a more validated 
questionnaire specific for PCOS subjects, the PCOS questionnaire, that involves analysis of emotions, hirsutism impact, weight, menstrual disorders, and infertility. ${ }^{138}$

In the literature, there is consistent evidence that patients with PCOS represent a group at high-risk for a lot of psychological disturbances and for reduced QoL. However, due to several biases and due to the heterogeneous nature of PCOS, with multiple phenotypes prevalence changing across the life span, it is difficult to establish from existing research as to what proportion of women with PCOS fall into the at-risk group. ${ }^{13}$ Moreover, the debate remains open about whether the increased prevalence of these psychological disorders is due to the PCOS itself or its features, such as obesity, hirsutism, irregular menses, and infertility. Relationship between obesity and QoL has been investigated, reporting an association between BMI and depression ${ }^{139}$ and poor QoL, ${ }^{134}$ with an improvement of depression and QoL score after weight loss. ${ }^{140,141}$ A recent meta-analysis ${ }^{136}$ is unable to perform a subanalysis of BMI-matched studies relative to association between anxiety symptoms and BMI, given the small numbers of studies included. Also the relationship between hyperandrogenism and anxiety symptoms need to be better evaluated because there are few studies evaluating this association and the available data are conflicting. ${ }^{136,142,143}$ Another potential factor that may contribute to worse QoL and/or mood disorders is the infertility and the unfulfilled wish for a child, but it has been suggested that the diagnosis and or treatments for infertility may result in depressive or anxiety symptoms but not in an increased risk of clinically significant psychological disorders. ${ }^{144}$

The high prevalence of depression and anxiety in these patients led many authors ${ }^{15,136,139}$ to include a psychological assessment not only in the initial evaluation of women with PCOS but also in their follow-up. Moreover, the ESHRE/ ASRM Consensus Group ${ }^{13}$ considers as premature a psychological screening to all women with PCOS. Nevertheless, more research should be addressed to the evaluation of the validity of the existing psychological questionnaire as screening tools and to the development of new appropriate screening instruments and interventions. ${ }^{13}$

\section{Management of early complications}

Several treatments for ovulatory infertility related to PCOS were proposed over the years. Table 1 summarizes the recommendations for the management of infertility in women with PCOS. On the other hand, only preliminary data are to date available regarding the management of obstetric complications in women with PCOS.

\section{Lifestyle modifications}

Obesity was closely related to IR; for this reason, weight loss represented a fundamental preliminary approach in the treatment of obese/overweight PCOS women. In fact, weight loss resulted in the restoration of spontaneous ovulation and higher fertility rate. ${ }^{145}$ An aggressive approach to reduce weight, including pharmacological strategies and the use of contraception and high-dose folic acid was proposed for obese women before planning a pregnancy. ${ }^{145}$

However, lifestyle modification programs are related to a low compliance and a high dropout rate. To overcome this problem, the feasibility and the efficacy of a structured exercise training program was evaluated in obese anovulatory PCOS patients. ${ }^{146}$ The structured exercise training resulted in a higher menses frequency and the ovulation in comparison with diet, even if the cumulative pregnancy rate was similar between two approaches. ${ }^{146}$ Successively, lifestyle modifications resulted more efficacious than clomiphene cictrate (CC) and metformin in terms of pregnancy rate, resulting in $12.2 \%, 14.4 \%, 14.8 \%$ and $20 \%$ of pregnancies after CC, metformin, CC plus metformin, and lifestyle modification, respectively. ${ }^{147}$

Recent guidelines suggested the use of exercise therapy in the management of overweight and obesity in PCOS. ${ }^{16}$ However, the effect of the weight loss on pregnancy outcome in women with PCOS has been not adequately assessed. Moreover, although confirmatory evidence-based data on the issue are lacking, we also consider the lifestyle modifications, including hypocaloric diet and physical activity, the first-line approaches for the prevention of early complications of PCOS in case of overweight and obesity.

\section{Clomiphene citrate}

Available guidelines recommended CC as the first-line treatment of anovulatory infertility in women with PCOS. ${ }^{16} \mathrm{CC}$ is an estrogen modulator, efficacious, simple to administer and to manage, safe and cheap. Specifically, CC was effective in increasing pregnancy rate compared to placebo (OR 5.8, 95\% CI 1.6-21.5). ${ }^{148}$

According to the standard protocol, $\mathrm{CC}$ is administered for 5 days from second or third day of the menstrual cycle, starting from $50 \mathrm{mg} /$ day and increasing up to $250 \mathrm{mg} /$ day. "Managed care" studies showed that the most effective dosage is $100-150 \mathrm{mg} /$ day and over $75 \%$ of ovulations occur already within a dosage of $100 \mathrm{mg} /$ day. ${ }^{149}$

Alternative protocols for $\mathrm{CC}$ administration were proposed in patients with known $\mathrm{CC}$ resistance. Extended $\mathrm{CC}$ regimen resulted in higher ovulation and pregnancy rates 
in comparison with gonadotropins ovulation induction, suggesting that in CC-resistant PCOS patients a further period of CC administration might be preferable. ${ }^{149}$

Further protocols for CC administration, such as luteal phase and stair-step regimens, were recently proposed. ${ }^{149}$ The administration of $100 \mathrm{mg}$ of CC daily in the luteal phase resulted in improved ovulation and pregnancy rates, although in any case the significance was obtained, whereas the total number of follicles during stimulation was significantly higher. ${ }^{149}$ Stair-step protocol, consisting in $50 \mathrm{mg} \mathrm{CC}$ for 5 days followed by weekly increasing dose of $50 \mathrm{mg} \mathrm{CC}$ in case of absent ovarian response, up to $150 \mathrm{mg}$ daily, resulted less time-consuming and more efficacious in terms of ovulation rate in CC-resistant women.

In our practice, $\mathrm{CC}$ (at a dosage of $100 \mathrm{mg} / \mathrm{day}$, and for not more than six cycles) remains a valid therapy for inducing ovulation in infertile PCOS patients.

\section{Aromatase inhibitors}

Aromatase inhibitors (AIs), such as estrogen modulators, were approved by the Food and Drug Administration as firstline adjuvant therapies for estrogen-receptor-positive breast cancer. ${ }^{150}$ Several data ${ }^{150}$ evaluated the efficacy of AIs, and in particular of letrozole, as first-line therapy for inducing ovulation in infertile patients affected by PCOS. Letrozole was usually administered at a dose of $2.5 \mathrm{mg} /$ day, for 5 days from the third to the seventh day of the menstrual cycle.

More recently, a meta-analysis of RCTs showed that letrozole is related to significantly higher live-birth rates than CC (OR 1.64, 95\% CI 1.32-2.04). ${ }^{151}$ Furthermore, the quality of the evidence was considered low due to the poor study transparency in reporting methods and in consideration of the more favorable results in the trials reporting live birth as primary end point. Excellent results of AIs in terms of cumulative live-birth rate and singleton pregnancy was reported in the largest multicenter randomized double-blind parallel controlled trial (Pregnancy in PCOS trial II, PPCOS II) comparing letrozole to $\mathrm{CC}{ }^{152}$

To date, letrozole remains an off-label drug. ${ }^{153}$ Studies are needed to validate the efficacy/safety of AIs over CC in further settings and to clarify its role in well-codified strategies and algorithms for ovulation induction in PCOS. ${ }^{150}$ Thus, the use of AIs in clinical and nonexperimental settings should be probably avoided.

\section{Metformin}

Metformin belongs to insulin-sensitizing drugs commonly used in treating T2DM. Because IR is a common condition in
PCOS women, metformin was introduced in clinical practice in the treatment of these patients. ${ }^{154}$

Metformin is available in two formulations: immediateand extended-release, which are administered orally. Even if no dose-finding study is available, metformin administration at incremental doses from 500 to $2,500 \mathrm{mg}$ /day was proposed to treat women with PCOS. ${ }^{154}$ Several systemic and local effects were demonstrated to explain the reproductive benefits due to metformin administration. ${ }^{154}$

In practice, metformin seemed to restore the ovulatory function after almost 3 months of treatment; thus it could be recommended in PCOS patients who absolutely wish to avoid multiple gestations and/or in patients who do not tolerate $\mathrm{CC}$ and/or in those who do not have an imminent reproductive need. ${ }^{154}$

Metformin seemed to have no benefit in terms of pregnancy and live-birth rates in comparison with $\mathrm{CC}$, even if it was effective in restoring ovulation in CC-resistant patients. ${ }^{154}$ Moreover, metformin addition to gonadotropinscontrolled ovarian stimulation for IVF and non-IVF cycles modulated the ovarian response to gonadotropins and reduced the risk for ovarian hyperstimulation syndrome (OHSS). Thus, its use could be planned for patients at high risk for OHSS. ${ }^{16,154}$ Finally, preliminary data suggested that metformin decreased the frequency of both early pregnancy loss and GDM, ${ }^{38}$ without increasing the risk for major birth defects in women with PCOS. ${ }^{155}$

To the present, metformin was still considered a drug of off-label use in women with PCOS. Guidelines ${ }^{16}$ discouraged the use of metformin as a first-line treatment for prevention of pregnancy complications, or for the treatment of obesity. No specific indication was given considering the potential effects of metformin pretreatment before starting subsequent standard infertility treatments. On the other hand, the use of metformin was suggested as an adjuvant therapy for infertility to prevent OHSS in women with PCOS undergoing IVF. ${ }^{156}$

On the basis of literature data and on our experience, metformin (at a dosage of 1,700-2,000 mg/day, and for at least three cycles) should be used in selected cases of oligoanovulatory PCOS patients who wish to avoid multiple gestation and/or in patients who do not tolerate $\mathrm{CC}$, are CC-resistant and/or in those who do not have an imminent reproductive need and/or in those undergoing IVF and at high-risk for OHSS (ie, PCOM phenotype).

\section{Laparoscopic ovarian drilling}

Laparoscopic ovarian drilling (LOD) is currently indicated as a safe, efficacious, and cost-effective alternative to 
gonadotropins for ovulation induction in infertile women with PCOS and who are CC-resistant. ${ }^{157}$ Advantages of LOD consisted in the absence of risk for OHSS and/or multiple pregnancies.

Since LOD improves ovarian responsiveness to $\mathrm{CC}$, it may be considered after $\mathrm{CC}$ failure before proceeding to the IVF. Despite its advantages, LOD should be considered a non-first-line therapy in PCOS due to the availability of valid, cheaper, and less invasive alternatives. ${ }^{157}$ Rather, it should be reserved to well-selected cases, ie, anovulatory CC-resistant PCOS women who are young and/or not compliant to the alternative treatments and/or needing laparoscopic assessment of the pelvis.

\section{Gonadotropins}

Gonadotropins are the cornerstone of the ovulation induction therapy in ovulatory infertility. However, gonadotropin administration was related to high costs, need for close monitoring, and high risk of multiple pregnancies and OHSS, particularly increased in PCOS subjects. For this reason, gonadotropins are often relegated as last treatment option in that population.

Notwithstanding, gonadotropins are used in ovulation induction protocols for many years, two main questions are still unanswered. The first regards the type of gonadotropins to use, while the last concerns the gonadotropin protocol to follow.

No difference between urinary $(\mathrm{u})$ and recombinant (r) follicular-stimulating hormone $(\mathrm{u} / \mathrm{rFSH})$ was observed in safety, ovulation rate, pregnancy, abortion, multiple pregnancy, and OHSS. ${ }^{158}$ Regarding the gonadotropins dose and regimen, efforts to reduce the frequency of OHSS have resulted in the development of low-dose protocols, which used a starting dose of 37.5 or $75 \mathrm{IU} /$ day instead the original conventional protocol. ${ }^{159}$ No difference was shown between the traditional "step-down" and the "low-dose step-up" regimen. ${ }^{159}$ On the contrary, the "low-dose" $\mathrm{rFSH}$ regimen was demonstrated to be more effective and welltolerated than uFSH in ovulation induction of CC-resistant PCOS patients. ${ }^{159}$ Finally, guidelines ${ }^{159}$ recommended a starting dose of gonadotropins of 37.5-50 IU/day, with small FSH dose increment of $50 \%$ of the initial or previous FSH dose in order to reduce the excessive ovarian stimulation.

In our practice, in PCOS patients, we use gonadotropins at low-dose, step-up protocol starting with a gonadotropin dose of 37.5 IU daily for 7 days; we usually increase the dose of $50 \%$ every week up to a total of 30 days. In case of no ovarian response, the maximal dosage achieved will be employed for the subsequent cycle(s).

\section{Management of the long-term complications}

The long-term risks observed in women with PCOS are not the same in all PCOS patients but they vary according to different phenotypes and results were negatively affected by obesity and lifestyle factors. When Rotterdam criteria are applied, almost uniformly accepted by the main scientific societies, ${ }^{13,15,16}$ the prevalence of PCOS in the population increases to over $20 \%,{ }^{160}$ with a large majority, approximately $75 \%$, of referred PCOS women having "classic" PCOS (according to NIH criteria) and the remaining 25\% equally divided between ovulatory and non-hyperandrogenic PCOS phenotypes. ${ }^{161}$ Several studies ${ }^{13,162}$ suggest that women with PCOS, based on the classic-NIH criteria, exhibit a more detrimental metabolic and cardiovascular profile compared to milder phenotypes. In line with these considerations, the main scientific societies dealing with PCOS, ${ }^{13,15,16,49}$ have recently proposed guidelines and consensus statements, suggesting a correct diagnostic approach to PCOS patients and an effort to properly identify the phenotype of each patient, with the aim to target specific treatments and to prevent these severe long-term risks.

In relation to metabolic risks, the ESHRE/ASRMSponsored PCOS Consensus Workshops Group ${ }^{13}$ and the AE-PCOS Society ${ }^{49}$ recommend performing an oral glucose tolerance test (OGTT), consisting of a fasting and a 2-hour glucose level using a $75 \mathrm{~g}$ oral glucose load, as screening for IGT and T2DM in PCOS women with classic-phenotype (hyperandrogenism and anovulation), obesity (BMI $>30 \mathrm{~kg} / \mathrm{m}^{2}$ ), acanthosis nigricans which represents a pathognomonic sign of IR, and a personal history of GDM or a family history of T2DM. The AE-PCOS Society ${ }^{49}$ recommends OGTT also in lean PCOS women with advanced age ( $>40$ years). The Endocrine Society and the European Society of Endocrinology ${ }^{16}$ broaden the OGTT recommendation to all adolescents and adult women with PCOS because considering all of them at high risk of IGT and T2DM. The major scientific societies ${ }^{13,15,16,49}$ converge to not use hemoglobin A1c as screening tool because it is not a sensitive methods of screening for T2DM in situations of risk, such as PCOS, ${ }^{163,164}$ and limited studies have shown poor sensitivity of hemoglobin A1c for detecting IGT, ${ }^{165}$ an important risk factor for CVD. ${ }^{166}$ Instead, there is no agreement about the interval for periodic follow-up of these patients. This interval varies from 2 years, or annually in those with IGT, according to AE-PCOS 
Society statement, ${ }^{49}$ to $3-5$ years based on Endocrine Society guideline; ${ }^{16}$ both agree to anticipate the interval in presence of risk factors, such as central adiposity, weight gain, and/or symptoms of diabetes develop.

In consideration of the increasing prevalence of obesity, particularly abdominal, and the important bearing on the phenotype of PCOS, it is recommended that BMI and waist circumference be determined at every visit, ${ }^{167}$ considering the presence of abdominal obesity in European women with a waist circumference of at least $80 \mathrm{~cm} .{ }^{167}$

Regarding the cardiovascular risk in PCOS women, AEPCOS Society ${ }^{49}$ proposed, according to CVD risk classification provided by American Heart Association, ${ }^{168}$ to categorize the PCOS-related CVD risk as: "at risk" for PCOS women with any following risk factors: obesity, cigarette smoking, hypertension, dyslipidemia, subclinical vascular disease, IGT, and/or family history of premature CVD ( $<55$ years of age in male relative, $<65$ years of age in female relative); and "at high risk" for PCOS women with metabolic syndrome and/or T2DM and/or overt vascular or renal disease.

There is an overall consensus about the increased cardiovascular risk in the PCOS patients, due to relevant metabolic dysfunctions. Therefore, all societes ${ }^{13,15,16,49}$ agree to recommend a CVD risk assessment at any age, for blood pressure, complete lipid profile (including total, LDL, HDL, non-HDL cholesterol, and triglycerides), waist circumference, BMI, glucose profile, cigarette smoking, and a family history of early CVD. Moreover, depression and anxiety disorders are recognized as risk factors for CVD and they are common in PCOS women; so it is suggested that PCOS patients be assessed also for depression, anxiety, and QoL. ${ }^{15,16,49}$ Because cardiovascular risk increases with age and it can be exacerbated by obesity and worsened by environmental insults, periodic reassessment for CVD risk is suggested but there is no agreement how often the CVD risk assessment should be repeated.

Finally, there is no agreement on the optimal method, whether ultrasound or endometrial biopsy, and timing of screening for endometrial cancer. Despite the established increased risk of endometrial cancer in PCOS women, ${ }^{118}$ there are no data supporting routine ultrasound screening for endometrial thickness ${ }^{169}$ or routine endometrial biopsy ${ }^{170}$ in asymptomatic women. In line with American Cancer Society Guidelines, ${ }^{171}$ the decision to assess for the presence of endometrial cancer should be based essentially on the presence of abnormal uterine bleeding or spotting. Other relevant decision factors are amenorrhea length, women's age, and the ultrasound appearance of endometrium. ${ }^{13}$
Lifestyle change, including hypocaloric diet and physical exercise, is considered a cornerstone of the management of women with PCOS presenting with obesity, particularly the abdominal phenotype, ${ }^{15}$ so it is generally recommended as a first-line approach for obese PCOS women. ${ }^{13,15,16,49}$ However, there are few randomized controlled trials that support this recommendation. A Cochrane review ${ }^{172}$ about the lifestyle's impact on PCOS women supports the benefits of lifestyle treatment in PCOS patients. In particular, when compared to minimal treatment (consisting of unstructured minimal dietary, exercise or behavioral advice), lifestyle intervention is able to improve anthropometric markers, such as weight and fat distribution, total testosterone levels, FerrimanGallwey score, fasting, and OGTT insulin concentrations. However, it does not reduce free androgen index that is considered a real marker of hyperandrogenism; it neither shows effects on OGTT glucose, fasting glucose levels, or lipid profile, compared to controls. ${ }^{172}$

The dietary advices represent the main components of the lifestyle changes, in particular for obese women, but the dietary composition seems to influence the metabolic improvements of the PCOS women to a lesser extent when compared to the weight loss. ${ }^{173}$ In fact, a 5\%-10\% weight loss is considered clinically significant and able to reduce IGT and metabolic syndrome prevalence in general population. ${ }^{174}$ However, no evidence-based data are actually available in order to suggest a specific hypocaloric diet. ${ }^{175} \mathrm{New}$ data, obtained in populations unselected for PCOS, seem to suggest a role for the Mediterranean diet for cardiometabolic prevention in PCOS patients, both obese and nonobese. ${ }^{175}$ A recent review and meta-analysis ${ }^{176}$ confirms that physical exercise and/or hypocaloric dieting are efficacious in overweight/obese women with PCOS through a significant, but small, effect on glucose and insulin blood levels. This review also found a direct correlation between the weight reduction and the improvement in metabolic parameters that could be attributed to decreased IR or to other factors, not allowing the evaluation of the independent effect of lifestyle modifications after accounting for weight loss. ${ }^{176}$ Also, the best type and optimal frequency of physical exercise required for treating PCOS has not been yet established. ${ }^{177}$ Other key issue, lifestyle treatment needs to be maintained long term for improvement of the metabolic PCOS outcomes but in literature exist only few randomized studies beyond 6 months of follow-up.

Currently, despite the limitations of the existing evidences, lifestyle changes, aiming at sustained weight loss, should be recommended as first-line treatment in overweight/ obese PCOS women, given the limited health risks, the low 
costs, and the modest benefits with other interventions. ${ }^{176}$ Future prospective requires longer and larger trials to draw stronger conclusions about the effects of lifestyle modifications on PCOS outcomes, to determine optimal weight loss for all these clinical improvements, and to establish the possible effects also in lean PCOS patients.

Since it is difficult to maintain a reduced weight for a long term with a correct lifestyle program, bariatric surgery in PCOS obese patients has been proposed as an effective tool for weight loss, ${ }^{178}$ and recent Australian guidelines on PCOS managment ${ }^{179}$ suggest lowering the BMI threshold to propose bariatric surgery, considering the great impact of obesity to metabolic impairment of these PCOS women. Hence, bariatric surgery could be considered as part of the treatment in obese PCOS patients ${ }^{15}$ but additional researches about the role of this surgery on the different aspects of the syndrome is required..$^{13}$

From a pharmacological standpoint, considering the metabolic and hormonal relevance of IR and associated compensatory hyperinsulinemia, common features of the PCOS women, the treatment choices have been expanded to insulin-sensitizing agents, in particular, metformin. ${ }^{154}$

The effects of metformin on obese PCOS patients are known since the first study of Velazquez et al. ${ }^{180}$ Since then, a large number of studies has been published to investigate the rationale of metformin use in PCOS women. The women with PCOS, both obese or nonobese, are characterized by an IR and hyperinsulinemia more relevant compared to that of age- and weight-matched control's women, suggesting a tendency toward IR which is independent of obesity. ${ }^{181}$ Accordingly, when an impaired insulin sensitivity is present, the use of metformin might be suggested. ${ }^{154,182} \mathrm{~A}$ Cochrane review ${ }^{183}$ showed that metformin reduces serum testosterone levels and fasting insulin concentrations to a significant extent only among nonobese women. However, metformin is unlikely to replace combined oral contraceptives as first-line therapy for hirsutism, as shown in another Cochrane review. ${ }^{184}$ Moreover, metformin is not of benefit in improving weight loss, insulin sensitivity, or lipid profiles; hence a long-term prophylactic treatment with metformin is unlikely to prevent progression to diabetes. ${ }^{183}$ In line with these considerations, the main Societies ${ }^{13,15,16}$ agree to considered metformin for prevention of diabetes in women with PCOS and IGT when lifestyle modification is not successful and/or as an adjuvant to general lifestyle modifications that remains the first-line therapy for PCOS women at increased metabolic risk.

Among the insulin-sensitizing agents, the thiazolidinediones (TZDs), including pioglitazone and rosiglitazone, are other insulin-sensitizing drugs that have been more recently studied in women with PCOS. They have been shown efficacy in improving insulin sensitivity and IGT, as well as ovulation rate and menstrual ciclicity, ${ }^{185,186}$ but they are not considered as a first-line choice for PCOS women because there is not sufficient evidence to support their metabolic superiority over metformin. ${ }^{185}$ In addition, TZDs are classified by FDA as category $\mathrm{C}$ (potential teratogenic risk in fertile women), they should not be prescribed to patients with any liver disease or liver biochemical abnormalities, and there is concern about possible adverse cardiovascular events associated with rosiglitazone use. ${ }^{187}$ Therefore, TZDs may be considered as an alternative therapy in insulin-resistant, obese PCOS patients who are intolerant or refractory to metformin, ${ }^{15}$ or in PCOS women with severe IR due to genetic disorder. ${ }^{188}$

At present, in overweight/obese PCOS women, we advice lifestyle changes (consisting of Mediterranean diet and physical activity) as preliminary approach, at any age, with the aim of weight loss. We suggest the association with metformin in obese/overweight PCOS patients when lifestyle program alone is not enough to obtain metabolic improvements and we proposed metformin use in lean PCOS patients with impaired insulin sensitivity.

\section{Conclusion}

Overall, women with PCOS show an increased risk of obstetric, cardiovascular, metabolic, and psychological complications compared to non-PCOS women. These risks do not have the same entity in all PCOS subjects and can change during life. This variability seems closely related to PCOS phenotypes. However, the pathogenetic mechanism which links PCOS to the high rate of early-term and long-term complications is not fully known. The same PCOS-related hormonal and metabolic features, such as hyperandrogenism, IR and related hyperinsulinemia, and visceral obesity, play a crucial role in increasing these risks but the exact mechanisms with which they act have not yet been completely elucidated.

PCOS is influenced by the race since ethnic differences occur in both the metabolic phenotype social models of behavior, likely contributing to the differing expressions of PCOS, with distinct impact on QoL of these women, as well as on the long-term consequences.

Despite the aging-related increase in IR and abdominal obesity in all women and the worsening of the cardiometabolic profile after the menopause, women with PCOS seem to improve or to reach a plateau in some metabolic risk factors during menopausal transition (eg, in LDL cholesterol levels ${ }^{189}$ 
or glucose tolerance ${ }^{64}$ ). At the moment, well-designed longitudinal follow-up studies comparing PCOS subjects with healthy women from early reproductive age into menopause are lacking, and those available provide unclear and conflicting findings. Indeed, the transition of women with PCOS into menopause and the potential PCOS phenotypes in postmenopause are poorly understood, even if an improved menstrual cyclicity and a substantial decrease in testosterone levels in PCOS women are observed.

In the light of these considerations, all women with a diagnosis of PCOS should be screened for the presence of obstetric, cardiovascular, and metabolic risk factors but it is equally important to consider the heterogeneity of the syndrome, due to age of patients and to several hormonal, reproductive, and ethnic features. Unfortunately, there are not yet available specific diagnostic criteria based on different age groups or based on specific ethnic origin. Further research is needed to improve diagnostic process with aim to select specific treatment, customizing the therapy and the lifestyle modifications.

\section{Author contributions}

All the authors provided a substantial contribution to the conception and design, acquisition of data, analysis and interpretation of data, revised the draft critically for intellectual content, and gave their approval for the final version of the manuscript.

\section{Disclosure}

The authors report no conflicts of interest in this work.

\section{References}

1. Azziz R, Woods KS, Reyna R, Key TJ, Knochenhauer ES, Yildiz BO. The prevalence and features of the polycystic ovary syndrome in an unselected population. J Clin Endocrinol Metab. 2004;89(6):2745-2749.

2. Kauffman RP, Baker TE, Baker VM, DiMarino P, Castracane D. Endocrine and metabolic differences among phenotypic expressions of polycystic ovary syndrome according to the 2003 Rotterdam consensus criteria. Am J Obstet Gynecol. 2008;198(6):670.e1-670.e10.

3. Diamanti-Kandarakis E, Kouli CR, Bergiele AT, et al. A survey of the polycystic ovary syndrome in the Greek island of Lesbos: hormonal and metabolic profile. J Clin Endocrinol Metab. 1999;84(11):4006-4011.

4. Asuncion M, Calvo RM, San Millan JL, Sancho J, Avila S, EscobarMorreale HF. A prospective study of the prevalence of the polycystic ovary syndrome in unselected Caucasian women from Spain. J Clin Endocrinol Metab. 2000;85(7):2434-2438.

5. March WA, Moore VM, Willson KJ, Phillips DI, Norman RJ, Davies MJ. The prevalence of polycystic ovary syndrome in a community sample assessed under contrasting diagnostic criteria. Hum Reprod. 2010;25(2):544-551.

6. Stein IF, Leventhal MI. Amenorrhea associated with bilateral polycystic ovaries. Am J Obstet Gynecol. 1935;29:181-191.

7. Zawadski JK, Dunaif A. Diagnostic criteria for polycystic ovary syndrome; toward a rational approach. In: Dunaif A, Givens JR, Haseltine FP, editors. Polycystic Ovary Syndrome. Boston MA: Blackwell Scientific; 1992:377-384.
8. Rotterdam ESHRE/ASRM-Sponsored PCOS Consensus Workshop Group. Revised 2003 consensus on diagnostic criteria and long-term health risks related to polycystic ovary syndrome (PCOS). Hum Reprod. 2004;19(1):41-47.

9. Goverde AJ, van Koert AJ, Eijkemans MJ, et al. Indicators for metabolic disturbances in anovulatory women with polycystic ovary syndrome diagnosed according to the Rotterdam consensus criteria. Hum Reprod. 2009;24(3):710-717.

10. Moran LJ, Misso ML, Wild RA, Norman RJ. Impaired glucose tolerance, type 2 diabetes and metabolic syndrome in polycystic ovary syndrome: a systematic review and meta-analysis. Hum Reprod Update. 2010;16(4):347-363.

11. Yildiz BO, Bozdag G, Yapici Z, Esinler I, Yarali H. Prevalence, phenotype and cardiometabolic risk of polycystic ovary syndrome under different diagnostic criteria. Hum Reprod. 2012;27(10):3067-3073.

12. Azziz R, Carmina E, Dewailly D, et al. Positions statement: criteria for defining polycystic ovary syndrome as a predominantly hyperandrogenic syndrome: an Androgen Excess Society guideline. J Clin Endocrinol Metab. 2006;91(11):4237-4245.

13. Fauser BC, Tarlatzis BC, Rebar RW, et al. Consensus on women's health aspects of polycystic ovary syndrome (PCOS): the Amsterdam ESHRE/ASRM-Sponsored 3rd PCOS Consensus Workshop Group. Fertil Steril. 2012;97(1):28-38.

14. National Institutes of Health (NIH). Evidence-based Methodology Workshop on Polycystic Ovary Syndrome. December 3-5, 2012. Available from: https://prevention.nih.gov/docs/programs/pcos/ finelreport.pdf

15. Conway G, Dewailly D, Diamanti-Kandarakis E, et al. The polycystic ovary syndrome: a position statement from the European Society of Endocrinology. Eur J Endocrinol. 2014;171(4):1-29.

16. Legro RS, Arslanian SA, Ehrmann DA, et al. Diagnosis and treatment of polycystic ovary syndrome: an endocrine society clinical practice guideline. J Clin Endocrinol Metab. 2013;98(12):4565-4592.

17. Orio F, Palomba S. Reproductive endocrinology: new guidelines for the diagnosis and treatment of PCOS. Nat Rev Endocrinol. 2014;10(3): 130-132.

18. Welt CK, Carmina E. Clinical review: lifecycle of polycystic ovary syndrome (PCOS): from in utero to menopause. J Clin Endocrinol Metab. 2013;98(12):4629-4638.

19. Hull MG. Epidemiology of infertility and polycystic ovarian disease: endocrinological and demographic studies. Gynecol Endocrinol. 1987;1:235-245.

20. Balen AH, Conway GS, Kaltsas G, et al. Polycystic ovary syndrome: the spectrum of the disorder in 1741 patients. Hum Reprod. 1995;10: 2107-2111.

21. Tian L, Shen H, Lu Q, Norman RJ, Wang J. Insulin resistance increases the risk of spontaneous abortion after assisted reproduction technology treatment. J Clin Endocrinol Metab. 2007;92(4):1430-1433.

22. Jungheim ES, Lanzendorf SE, Odem RR, Moley KH, Chang AS, Ratts VS. Morbid obesity is associated with lower clinical pregnancy rates after in vitro fertilization in women with polycystic ovary syndrome. Fertil Steril. 2009;92(1):256-261.

23. Jakubowicz DJ, Essah PA, Seppälä M, et al. Reduced serum glycodelin and insulin-like growth factor-binding protein-1 in women with polycystic ovary syndrome during first trimester of pregnancy. J Clin Endocrinol Metab. 2004;89(2):833-839.

24. Palomba S, Russo T, Orio F Jr. Uterine effects of clomiphene citrate in women with polycystic ovary syndrome: a prospective controlled study. Hum Reprod. 2006;21(11):2823-2829.

25. Palomba S, Orio F Jr, Falbo A, Russo T, Tolino A, Zullo F. Effects of metformin and clomiphene citrate on ovarian vascularity in patients with polycystic ovary syndrome. Fertil Steril. 2006;86(6):1694-1701.

26. Teissier MP, Chable H, Paulhac S, Aubard Y. Comparison of follicle steroidogenesis from normal and polycystic ovaries in women undergoing IVF: relationship between steroid concentrations, follicle size, oocyte quality and fecundability. Hum Reprod. 2000;15(12): 2471-2477. 
27. Palomba S, Falbo A, Russo T, Orio F, Tolino A, Zullo F. Systemic and local effects of metformin administration in patients with polycystic ovary syndrome (PCOS): relationship to the ovulatory response. Hum Reprod. 2010;25(4):1005-1013.

28. Wood JR, Dumesic DA, Abbott DH, Strauss JF 3rd. Molecular abnormalities in oocytes from women with polycystic ovary syndrome revealed by microarray analysis. J Clin Endocrinol Metab. 2007;92(2): 705-713.

29. Dahlgren E, Johansson S, Lindstedt G, et al. Women with polycystic ovary syndrome wedge resected in 1956 to 1965: a long-term follow-up focusing on natural history and circulating hormones. Fertil Steril. 1992;57(3):505-513

30. Cibula D, Cifkova R, Fanta M, Poledne R, Zivny J, Skibova J. Increased risk of non-insulin dependent diabetes mellitus, arterial hypertension and coronary artery disease in perimenopausal women with a history of the polycystic ovary syndrome. Hum Reprod. 2000;15:785-789.

31. Wild S, Pierpoint T, McKeigue P, Jacobs H. Cardiovascular disease in women with polycystic ovary syndrome at long-term follow-up a retrospective cohort study. Clin Endocrinol (Oxf). 2000;52:595-600.

32. Hudecova M, Holte J, Olovsson M, Sundström Poromaa I. Long-term follow-up of patients with polycystic ovary syndrome: reproductive outcome and ovarian reserve. Hum Reprod. 2009;24(5):1176-1183.

33. Korhonen S, Hippelainen M, Vanhala M, Heinonen S, Niskanen L. The androgenic sex hormone profile is an essential feature of metabolic syndrome in premenopausal women: a controlled community-based study. Fertil Steril. 2003;79:1327-1334.

34. Elting MW, Korsen TJ, Rekers-Mombarg LT, Schoemaker J. Women with polycystic ovary syndrome gain regular menstrual cycles when ageing. Hum Reprod. 2000;15:24-28.

35. Kalra SK, Ratcliffeb SJ, Dokrasn A. Is the fertile windows extended in women with polycystic ovary syndrome? Utilizing the Society for Assisted Reproductive Technology registry to assess the impact of reproductive aging on live-birth rate. Fertil Steril. 2013;100(1):208-213.

36. HeijnenEM,Eijkemans MJ,HughesEG, Laven JS, MacklonNS, FauserBC A meta-analysis of outcomes of conventional IVF in women with polycystic ovary syndrome. Hum Reprod Update. 2006;12(1):13-21.

37. Rotterdam ESHRE/ASRM-Sponsored PCOS Consensus Workshop Group. Revised 2003 consensus on diagnostic criteria and longterm health risks related to polycystic ovary syndrome. Fertil Steril 2004;81(1):19-25.

38. Palomba S, de Wilde MA, Falbo A, Koster MPH, La Sala GB, Fauser CJM. Pregnancy complications in women with polycystic ovary syndrome: new clinical and pathophysiological insights. Hum Reprod Update. 2015 Jun 27. pii: dmv029.

39. Boomsma CM, Eijkemans MJ, Hughes EG, Visser GH, Fauser BC, Macklon NS. A meta-analysis of pregnancy outcomes in women with polycystic ovary syndrome. Hum Reprod Update. 2006;12(6): 673-683.

40. Kjerulff LE, Sanchez-Ramos L, Duffy D. Pregnancy outcomes in women with polycystic ovary syndrome: a metaanalysis. Am J Obstet Gynecol. 2011;204(6):558.e1-558.e6.

41. Qin JZ, Pang LH, Li MJ, Fan XJ, Huang RD, Chen HY. Obstetric complications in women with polycystic ovary syndrome: a systematic review and meta-analysis. Reprod Biol Endocrinol. 2013;26:11-56.

42. Liu L, Tong X, Jiang L, Li TC, Zhou F, Zhang S. A comparison of the miscarriage rate between women with and without polycystic ovarian syndrome undergoing IVF treatment. Eur J Obstet Gynecol Reprod Biol. 2014;176(4):178-182.

43. Palomba S, Chiossi G, Falbo A, et al. Low-grade chronic inflammation in pregnant women with polycystic ovary syndrome. J Clin Endocrinol Metab. 2014;99(8):2942-2951.

44. Palomba S, Falbo A, Chiossi G, et al. Lipid profile in pregnant women with polycystic ovary syndrome. Steroids. 2014;88:36-43.

45. de Wilde MA, Veltman-Verhulst SM, Goverde AJ, et al. Preconception predictors of gestational diabetes: a multicentre prospective cohort study on the predominant complication of pregnancy in polycystic ovary syndrome. Hum Reprod. 2014;29(6):1327-1236.
46. Naver KV, Grinsted J, Larsen SO, et al. Increased risk of preterm delivery and pre-eclampsia in women with polycystic ovary syndrome and hyperandrogenaemia. BJOG. 2014;121(5):575-581.

47. Han AR, Kim HO, Cha SW, et al. Adverse pregnancy outcomes with assisted reproductive technology in non-obese women with polycystic ovary syndrome: a case-control study. Clin Exp Reprod Med. 2011;38(2): $103-108$.

48. Roos N, KielerH, SahlinL, Ekman-Ordeberg G, FalconerH, StephanssonO. Risk of adverse pregnancy outcomes in women with polycystic ovary syndrome: population based cohort study. BMJ. 2011;343: d6309.

49. Wild RA, Carmina E, Diamanti-Kandarakis E, et al. Assessment of cardiovascular risk and prevention of cardiovascular disease in women with the Polycystic Ovary Syndrome: a consensus statement by the Androgen Wxcess and Polycystic Ovary Syndrome (AE-PCOS) Society. J Clin Endocrinol Metab. 2010;95(2):2038-2049.

50. Toulis KA, Goulis DG, Mintziori G, et al. Meta-analysis of cardiovascular disease risk markers in women with polycystic ovary syndrome. Hum Reprod Update. 2011;17(6):741-760.

51. Yusuf S, Hawken S, Ounpuu S, et al. Effect of potentially modifiable risk factors associated with myocardial infarction in 52 countries (the INTERHEART study): case-control study. Lancet. 2004;364(9438): 937-952.

52. Martins WdeP, Soares GM, Vieira CS, dos Reis RM, de Sá MF, Ferriani RA. Cardiovascular risk markers in polycystic ovary syndrome in women with and without insulin resistance. Rev Bras Ginecol Obstet. 2009;31(3):111-116.

53. Scicchitano P, Dentamaro I, Carbonara R, et al. Cardiovascular risk in women with PCOS. Int J Endocrinol Metab. 2012;10(4):611-618.

54. Chen MJ, Yang WS, Yang JH, Chen CL, Ho HN, Yang YS. Relationship between androgen levels and blood pressure in young women with polycystic ovary syndrome. Hypertension. 2007;49(6):1442-1447.

55. Holte J, Gennarelli G, Berne C, Bergh T, Lithell H. Elevated ambulatory day-time blood pressure in women with polycystic ovary syndrome: a sign of a pre-hypertensive state? Hum Reprod. 1996;11(1):23-28.

56. Legro RS, Kunselman AR, Dunaif A. Prevalence and predictors of dyslipidemia in women with polycystic ovary syndrome. Am J Med. 2001;111(8):607-613.

57. Essah PA, Nestler JE, Carmina E. Differences in dyslipidemia between American and Italian women with polycystic ovary syndrome. J Endocrinol Invest. 2008;31(1):35-41.

58. Austin MA, King MC, Vranizan KM, Krauss RM. Atherogenic lipoprotein phenotype. A proposed genetic marker for coronary heart disease risk. Circulation. 1990;82(2):495-506.

59. Rizzo M, Berneis K. Lipid triad or atherogenic lipoprotein phenotype: a role in cardiovascular prevention? J Atheroscler Thromb. 2005;12(5): 237-239.

60. Brunzell JD, Ayyobi AF. Dyslipidemia in the metabolic syndrome and type 2 diabetes mellitus. Am J Med. 2003;115:24S-28S.

61. Valkenburg O, Steegers-Theunissen RP, Smedts HP, et al. A more atherogenic serum lipoprotein profile is present in women with polycystic ovary syndrome: a case-control study. J Clin Endocrinol Metab. 2008;93(2):470-476.

62. Zhang J, Fan P, Liu H, Bai H, Wang Y, Zhang F. Apolipoprotein A-I and B levels, dyslipidemia and metabolic syndrome in south-west Chinese women with PCOS. Hum Reprod. 2012;27(8):2484-2493.

63. Joharatnam J, Barber TM, Webber L, Conway GS, McCarthy MI, Franks S. Determinants of dyslipidaemia in probands with polycystic ovary syndrome and their sisters. Clin Endocrinol (Oxford). 2011;74(6): 714-719.

64. Legro RS, Gnatuk CL, Kunselman AR, Dunaif A. Changes in glucose tolerance over time in women with polycystic ovary syndrome: a controlled study. J Clin Endocrinol Metab. 2005;90(6):3236-3242.

65. Norman RJ, Masters L, Milner CR, Wang JX, Davies MJ. Relative risk of conversion from normoglycaemia to impaired glucose tolerance or non-insulin dependent diabetes mellitus in polycystic ovarian syndrome. Hum Reprod. 2001;16(9):1995-1998. 
66. Wang ET, Calderon-Margalit R, Cedars MI, et al. Polycystic ovary syndrome and risk for long-term diabetes and dyslipidemia. Obstet Gynecol. 2011;117(1):6-13.

67. Pugliese D, Nicoletti G, Andreula C, Ciccone M, Triggiani G. Combined protein $\mathrm{C}$ deficiency and protein $\mathrm{C}$ activated resistance as a cause of caval, peripheral, and cerebral venous thrombosis - a case report. Angiology. 1998;49(5):399-401.

68. Ridker PM. Clinical application of C-reactive protein for cardiovascular disease detection and prevention. Circulation. 2003;107(3):363-369.

69. Oh JY, Lee JA, Lee H, Oh JY, Sung YA, Chung H. Serum C-reactive protein levels in normal-weight polycystic ovary syndrome. Korean $J$ Intern Med. 2009;24(4):350-355.

70. Möhlig M, Spranger J, Osterhoff M, et al. The polycystic ovary syndrome per se is not associated with increased chronic inflammation. Eur J Endocrinol. 2004;150(4):525-532.

71. Yarali H, Yildirir A, Aybar F, et al. Diastolic dysfunction and increased serum homocysteine concentrations may contribute to increased cardiovascular risk in patients with polycystic ovary syndrome. Fertil Steril. 2001;76(3):511-516.

72. Schachter M, Raziel A, Friedler S, Strassburger D, Bern O, Ron-El R. Insulin resistance in patients with polycystic ovary syndrome is associated with elevated plasma homocysteine. Hum Reprod. 2003; 18(4):721-727.

73. Loverro G, Lorusso F, Mei L, Depalo R, Cormio G, Selvaggi L. The plasma homocysteine levels are increased in polycystic ovary syndrome. Gynecol Obstet Invest. 2002;53(3):157-162.

74. Wiltgen D, Spritzer PM. Variation in metabolic and cardiovascular risk in women with different polycystic ovary syndrome phenotypes. Fertil Steril. 2010;94(6):2493-2496.

75. Samy N, Hashim M, Sayed M, Said M. Clinical significance of inflammatory markers in polycystic ovary syndrome: their relationship to insulin resistance and body mass index. Dis Markers. 2009;26(4):163-170.

76. Elting MW, Korsen TJ, Schoemaker J. Obesity, rather than menstrual cycle pattern or follicle cohort size, determines hyperinsulinaemia, dyslipidaemia and hypertension in ageing women with polycystic ovary syndrome. Clin Endocrinol (Oxf). 2001;55(6):767-776.

77. Wild S, Pierpoint T, Jacobs H, McKeigue P. Long-term consequences of polycystic ovary syndrome: results of a 31 year follow-up study. Hum Fertil (Camb). 2000;3(2):101-105.

78. Wild RA, Grubb B, Hartz A, Van Nort JJ, Bachman W, Bartholomew M. Clinical signs of androgen excess as risk factors for coronary artery disease. Fertil Steril. 1990;54(2):255-259.

79. Birdsall MA, Farquhar CM, White HD. Association between polycystic ovaries and extent of coronary artery disease in women having cardiac catheterization. Ann Intern Med. 1997;126(1):32-35.

80. Dokras A. Cardiovascular disease risk in women with PCOS. Steroids. 2013;78(8):773-776.

81. Sprung VS, Atkinson G, Cuthbertson DJ, et al. Endothelial function measured using flow-mediated dilation in polycystic ovary syndrome: a meta-analysis of the observational studies. Clin Endocrinol (Oxf). 2013;78(3):438-446.

82. Paradisi G, Steinberg HO, Hempfling A, et al. Polycystic ovary syndrome is associated with endothelial dysfunction. Circulation. 2001;103(10):1410-1415.

83. Kravariti M, Naka KK, Kalantaridou SN, et al. Predictors of endothelial dysfunction in young women with polycystic ovary syndrome. J Clin Endocrinol Metab. 2005;90(9):5088-5095.

84. Meyer ML, Malek AM, Wild RA, Korytkowski MT, Talbott EO. Carotid artery intima-media thickness in polycystic ovary syndrome: a systematic review and meta-analysis. Hum Reprod Update. 2012;18(2):112-126.

85. Arad Y, Spadaro LA, Goodman K, Newstein D, Guerci AD. Prediction of coronary events with electron beam computed tomography. $J \mathrm{Am}$ Coll Cardiol. 2000;36(4):1253-1260.

86. Christian RC, Dumesic DA, Behrenbeck T, Oberg AL, Sheedy PF, Fitzpatrick LA. Prevalence and predictors of coronary artery calcification in women with polycystic ovary syndrome. J Clin Endocrinol Metab. 2003;88(6):2562-2568.
87. TalbottEO, GuzickDS, Sutton-Tyrrell K, Hugh-Pemu KP,ZborowskiJV, Remsberg KE, et al. Evidence for association between polycystic ovary syndrome and premature carotid atherosclerosis in middle-aged women. Arterioscler Thromb Vasc Biol. 2000;20(11):2414-2421.

88. Luque-Ramirez M, Mendieta-Azcona C, Alvarez-Blasco F, EscobarMorreale HF. Androgen excess is associated with the increased carotid intima-media thickness observed in young women with polycystic ovary syndrome. Hum Reprod. 2007;22(12):3197-3203.

89. Talbott EO, Zborowski J, Rager J, Stragand JR. Is there an independent effect of polycystic ovary syndrome (PCOS) and menopause on the prevalence of subclinical atherosclerosis in middle aged women? Vasc Health Risk Manag. 2008;4(2):453-462.

90. Schmidt J, Landin-Wilhelmsen K, Brännström M, Dahlgren E. Cardiovascular disease and risk factors in PCOS women of postmenopausal age: a 21-year controlled follow-up study. J Clin Endocrinol Metab. 2011;96(12):3794-3803.

91. De Groot PC, Dekkers OM, Romijn JA, Dieben SW, Helmerhorst FM. PCOS, coronary heart disease, stroke and the influence of obesity: a systematic review and meta-analysis. Hum Reprod Update. 2011;17(4):495-500.

92. Anderson SA, Barry JA, Hardiman PJ. Risk of coronary heart disease and risk of stroke in women with polycystic ovary syndrome: a systematic review and meta-analysis. Int J Cardiol. 2014;176(2):486-487.

93. Lim SS, Davies MJ, Norman RJ, Moran LJ. Overweight, obesity and central obesity in women with polycystic ovary syndrome: a systematic review and meta-analysis. Hum Reprod Update. 2012;18(6): 618-637.

94. Ching HL, Burke V, Stuckey BG. Quality of life and psychological morbidity in women with polycystic ovary syndrome: body mass index, age and the provision of patient information are significant modifiers. Clin Endocrinol (Oxf). 2007;66(3):373-379.

95. Glueck CJ, Dharashivkar S, Wang P, et al. Obesity and extreme obesity, manifest by ages 20-24 years, continuing through 32-41 years in women, should alert physicians to the diagnostic likelihood of polycystic ovary syndrome as a reversible underlying endocrinopathy. Eur J Obstet Gynecol Reprod Biol. 2005;122(2):206-212.

96. Chen X, Ni R, Mo Y, Li L, Yang D. Appropriate BMI levels for PCOS patients in Southern China. Hum Reprod. 2010;25(5):1295-1302.

97. Stepto NK, Cassar S, Joham AE, et al. Women with polycystic ovary syndrome have intrinsic insulin resistance on euglycaemichyperinsulaemic clamp. Hum Reprod. 2013;28(3):777-784.

98. Teede HJ, Joham AE, Paul E, et al. Longitudinal weight gain in women identified with polycystic ovary syndrome: results of an observational study in young women. Obesity. 2013;21(8):1526-1532.

99. Bates GW, Legro RS. Long-term management of Polycystic Ovarian Syndrome (PCOS). Mol Cel Endocrinol. 2013;373(1-2):91-97.

100. Carmina E, Bucchieri S, Esposito A, et al. Abdominal fat quantity and distribution in women with polycystic ovary syndrome and extent of its relation to insulin resistance. J Clin Endocrinol Metab. 2007;92(7):2500-2505.

101. Cascella T, Palomba S, De Sio I, et al. Visceral fat is associated with cardiovascular risk in women with polycystic ovary syndrome. Hum Reprod. 2008;23(1):153-159.

102. Lord J, Thomas R, Fox B, Acharya U, Wilkin T. The central issue? Visceral fat mass is a good marker of insulin resistance and metabolic disturbance in women with polycystic ovary syndrome. BJOG. 2006;113(10):1203-1209.

103. Pasquali R, Casimirri F, Venturoli S, et al. Body fat distribution has weight-independent effects on clinical, hormonal, and metabolic features of women with polycystic ovary syndrome. Metabolism. 1994;43(6):706-713.

104. Vrbikova J, Hainer V. Obesity and polycystic ovary syndrome. Obes Facts. 2009;2(1):26-35.

105. Sam S, Dunaif A. Polycystic ovary syndrome: syndrome XX? Trends Endocrinol Metab. 2003;14(8):365-370.

106. Diamanti-Kandarakis E, Dunaif A. Insulin resistance and the polycystic ovary syndrome revisited: an update on mechanisms and implications. Endocr Rev. 2012;33(6):981-1030. 
107. Tosi F, Di Sarra D, Kaufman JM, et al. Total body fat and central fat mass independently predict insulin resistance but not hyperandrogenemia in women with polycystic ovary syndrome. J Clin Endocrinol Metab. 2015;100(2):661-669.

108. Alberti KG, Zimmet P, Shaw J. International Diabetes Federation: a consensus on type 2 diabetes prevention. Diabet Med. 2007; 24(5):451-463.

109. Gambineri A, Patton L, Altieri P, et al. Polycystic ovary syndrome is a risk factor for type 2 diabetes: results from a long-term prospective study. Diabetes. 2012;61(9):2369-2374.

110. Daan NM, Louwers YV, Koster MP, et al. Cardiovascular and metabolic profiles amongst different polycystic ovary syndrome phenotypes: who is really at risk? Fertil Steril. 2014;102(5):1444-1451.

111. Apridonidze T, Essah PA, Iuorno MJ, Nestler JE. Prevalence and characteristics of the metabolic syndrome in women with polycystic ovary syndrome. J Clin Endocrinol Metab. 2005;90(4): 1929-1935.

112. Jovanovic VP, Carmina E, Lobo RA. Not all women diagnosed with PCOS share the same cardiovascular risk profiles. Fertil Steril. 2010;94(3):826-832.

113. Moran L, Teede H. Metabolic features of the reproductive phenotypes of polycystic ovary syndrome. Hum Reprod Update. 2009; $15(4): 477-488$

114. Moran LJ, Norman RJ, Teede HJ. Metabolic risk in PCOS: phenotype and adiposity impact. Trends Endocrinol Metab. 2015; 26(3):136-143

115. Moran LJ, Strauss BJ, Teede HJ. Diabetes risk score in the diagnostic categories of polycystic ovary syndrome. Fertil Steril. 2011;95(5):1742-1748

116. Hardiman P, Pillay OC, Atiomo W. Polycystic ovary syndrome and endometrial carcinoma. Lancet. 2003;361(9371):1810-1812.

117. Genazzani AR, Gadducci A, Gambacciani M. Controversial issues in climacteric medicine II. Hormone replacement therapy and cancer International Menopause Society Expert Workshop. Climacteric. 2001;4(3):181-193.

118. Barry JA, Azizia MM, Hardiman PJ. Risk of endometrial, ovarian and breast cancer in women with polycystic ovary syndrome: a systematic review and meta-analysis. Hum Reprod Update. 2014; 20(5):748-758

119. Broekmans FJ, Knauff EAH, Valkenburg O, Laven JS, Eijkemans MJ, Fauser BCJM. PCOS according to the Rotterdam consensus criteria: change in prevalence among WHO-II anovulation and association with metabolic factors. BJOG. 2006;113(10):1210-1217.

120. Haoula Z, Salman M, Atiomo W. Evaluating the association between endometrial cancer and polycystic ovary syndrome. Hum Reprod. 2012;27(5):1327-1331.

121. Chittenden BG, Fullerton G, Maheshwari A, Bhattacharya S. Polycystic ovary syndrome and the risk of gynaecological cancer: a systematic review. Reprod Biomed Online. 2009;19(3):398-405.

122. Giovannucci E. Metabolic syndrome, hyperinsulinemia, and colon cancer: a review. Am J Clin Nutr. 2007;86(3):s836-s842.

123. Iatrakis $\mathrm{G}$, Zervoudis S, Saviolakis A, et al. Women younger than 50 years with endometrial cancer. Eur J Gynaecol Oncol. 2006;27(4): 399-400.

124. Zucchetto A, Serraino D, Polesel J, et al. Hormone-related factors and gynecological conditions in relation to endometrial cancer risk. Eur J Cancer Prev. 2009;18(4):316-321.

125. Niwa K, Imai A, Hashimoto M, et al. A case-control study of uterine endometrial cancer of pre- and post-menopausal women. Oncol Rep 2000;7(1):89-93.

126. Dowling RJ, Goodwin PJ, Stambolic V. Understanding the benefit of metformin use in cancer treatment. MBC Med. 2011;9:33.

127. Engelman JA, Cantley LC. Chemoprevention meets glucose control. Cancer Prev Res. 2010;9(9):1049-1052.

128. Wang YL, Xie Y, Yu L, et al. Metformin promotes progesterone receptor expression via inhibition of mammalian target of rapamycin (mTOR) in endometrial cancer cells. J Steroid Biochem Mol Biol. 2011;126(3-5):113-120.
129. Legro RS, Zaino RJ, Demers LM, et al. The effects of metformin and rosiglitazone, alone and in combination, on the ovary and endometrium in polycystic ovary syndrome. Am J Obstet Gynecol. 2007; 196(4):402.

130. Shafiee MN, Khan G, Ariffin R, et al. Preventing endometrial cancer risk in polycystic ovarian syndrome (PCOS) women: could metformin help? Gynecol Oncol. 2014;132(1):248-253.

131. Pierpoint T, McKeigue PM, Isaacs AJ, Wild SH, Jacobs HS. Mortality of women with polycystic ovary syndrome at long-term follow-up. J Clin Epidemiol. 1998;51(7):581-586.

132. Dokras A, Clifton S, Futterweit W, Wild R. Increased risk for abnormal depression scores in women with polycystic ovary syndrome: a systematic review and meta-analysis. Obstet Gynecol. 2011;117(1):145-152.

133. Coffey S, Bano G, Mason H. Health-related quality of life in women with PCOS: a comparison with the general population using the PCOS questionnaire and the SF-36. Gynecol Endocrinol. 2006;22(2):80-86.

134. Barnard L, Ferriday D, Guenther N, Strauss B, Balen AH, Dye L. Quality of life and psychological wellbeing in polycystic ovary syndrome. Hum Reprod. 2007;22(8):2279-2286.

135. Moran LJ, Deeks AA, Gibson-Helm ME, Teede HJ. Psychological parameters in the reproductive phenotypes of polycystic ovary syndrome. Hum Reprod. 2012;27(7):2082-2088.

136. Dokras A, Clifton S, Futterweit W, Wild R. Increased prevalence of anxiety symptoms in women with polycystic ovary syndrome: systematic review and meta-analysis. Fertil Steril. 2012;97(1):225-230.

137. Laggari V, Diareme S, Christogiorgos S, et al. Anxiety and depression in adolescents with polycystic ovary syndrome and MayerRokitansky-Kuster-Hauser syndrome. J Psychosom Obstet Gynaecol. 2009;30(2):83-88.

138. Jones G, Benes K, Clark T, et al. The PCOS health-related quality of life questionnaire (PCSOQ): a validation. Hum Reprod. 2004;19(2):371-377.

139. Barry JA, Kuczmierczyk AR, Hardiman PJ. Anxiety and depression in polycystic ovary syndrome: a systematic review and meta-analysis. Hum Reprod. 2011;26(9):2442-2451.

140. Harris-Glocker M, Davidson K, Kochman L, Guzick D, Hoeger K. Improvement in quality-of-life questionnaire measures in obese adolescent females with polycystic ovary syndrome treated with lifestyle changes and oral contraceptives, with or without metformin. Fertil Steril. 2010;93(3):1016-1019.

141. Thomson RL, Buckley JD, Lim SS, et al. Lifestyle management improves quality of life and depression in overweight and obese women with polycystic ovary syndrome. Fertil Steril. 2010;94(5):1812-1816.

142. Jedel E, Gustafson D, Waern M, et al. Sex steroids, insulin sensitivity and sympathetic nerve activity in relation to affective symptoms in women with polycystic ovary syndrome. Psychoneuroendocrinology. 2011;36(10):1470-1479.

143. Weiner CL, Primeau M, Ehrmann DA. Androgens and mood dysfunction in women: comparison of women with PCOS to healthy controls. Psychosom Med. 2004;66(3):356-362.

144. Mansson M, Holte J, Landin-Wilhelmsen K, Dahlgren E, Johansson A, Landen $\mathrm{M}$. Women with polycystic ovary syndrome are often depressed or anxious - a case control study. Psychoneuroendocrinology. 2008;33(8):1132-1138.

145. Orio F, Tafuri D, Ascione A, et al. Lifestyle changes in the management of adulthood and childhood obesity. Minerva Endocrinol. 2014.

146. Palomba S, Giallauria F, Falbo A, et al. Structured exercise training programme versus hypocaloric hyperproteic diet in obese polycystic ovary syndrome patients with anovulatory infertility: a 24-week pilot study. Hum Reprod. 2008;23(3):642-650.

147. Palomba S, Falbo A, Giallauria F, et al. Six weeks of structured exercise training and hypocaloric diet increases the probability of ovulation after clomiphene citrate in overweight and obese patients with polycystic ovary syndrome: a randomized controlled trial. Hum Reprod. 2010;25(11):2783-2791. 
148. Brown J, Farquhar C, Beck J, Boothroyd C, Hughes E. Clomiphene and anti-oestrogens for ovulation induction in PCOS [review]. Cochrane Database Syst Rev. 2009;7(4):CD002249.

149. Palomba S, Falbo A, Zullo F. Management strategies for ovulation induction in women with polycystic ovary syndrome and known clomifene citrate resistance. Curr Opin Obstet Gynecol. 2009;21(6): 465-473.

150. Palomba S. Aromatase inhibitors for ovulation induction. J Clin Endocrinol Metab. 2015;100(5):1742-1747.

151. Franik S, Kremer JA, Nelen WL, Farquhar C. Aromatase inhibitors for subfertile women with polycystic ovary syndrome. Cochrane Database Syst Rev. 2014;2(1):CD010287.

152. Legro RS, Zhang H; Eunice Kennedy Shriver NICHD Reproductive Medicine Network. Letrozole or clomiphene for infertility in the polycystic ovary syndrome. $N$ Engl J Med. 2014;371(15):1463-1464.

153. Vitek W, Alur S, Hoeger KM. Off-label drug use in the treatment of polycystic ovary syndrome. Fertil Steril. 2015;103(3):605-611.

154. Palomba S, Falbo A, Zullo F, Orio F Jr. Evidence-based and potential benefits of metformin in the polycystic ovary syndrome: a comprehensive review. Endocr Rev. 2009;30(1):1-50.

155. Cassina $\mathrm{M}$, Donà $\mathrm{M}$, Di Gianantonio E, Litta $\mathrm{P}$, Clementi $\mathrm{M}$. First-trimester exposure to metformin and risk of birth defects: a systematic review and meta-analysis. Hum Reprod Update. 2014; 20(5):656-669.

156. Palomba S, Falbo A, La Sala GB. Effects of metformin in women with polycystic ovary syndrome treated with gonadotrophins for in vitro fertilisation and intracytoplasmic sperm injection cycles: a systematic review and meta-analysis of randomised controlled trials. BJOG. 2013;120(3):267-276.

157. Mitra S, Nayak PK, Agrawal S. Laparoscopic ovarian drilling: an alternative but not the ultimate in the management of polycystic ovary syndrome. J Nat Sci Biol Med. 2015;6(1):40-48.

158. Bayram N, van Wely M, van Der Veen F. Recombinant FSH versus urinary gonadotrophins or recombinant FSH for ovulation induction in subfertility associated with polycystic ovary syndrome. Cochrane Database Syst Rev. 2001;1(2):CD002121.

159. Thessaloniki ESHRE/ASRM-Sponsored PCOS Consensus Workshop Group. Consensus on infertility treatment related to polycystic ovary syndrome. Hum Reprod. 2008;23(3):462-477.

160. Belosi C, Selvaggi L, Apa R, et al. Is the PCOS diagnosis solved by ESHRE/ASRM 2003 consensus or could it include ultrasound examination of the ovarian stroma? Hum Reprod. 2006;21(12):3108-3115.

161. Abbott DH, Tarantal AF, Dumesic DA. Fetal, infant, adolescent and adult phenotypes of polycystic ovary syndrome in prenatally androgenized female rhesus monkeys. Am J Perinatol. 2009; 71(9): 776-784.

162. Ehrmann DA. Polycystic ovary syndrome. NEngl JMed. 2005;352(12): 1223-1236.

163. Hurd WW, Abdel-Rahman MY, Ismail SA, Abdellah MA, SchmotzerCL, Sood A. Comparison of diabetes mellitus and insulin resistance screening methods for women with polycystic ovary syndrome. Fertil Steril. 2011;96(4):1043-1047.

164. Lerchbaum E, Schwetz V, Giuliani A, Obermayer-Pietsch B. Assessment of glucose metabolism in polycystic ovary syndrome: HbA1c or fasting glucose compared with the oral glucose tolerance test as a screening method. Hum Reprod. 2013;28(9):2537-2544.

165. Velling Magnussen L, Mumm H, Andersen M, Glintborg D. Hemoglobin A1c as a tool for the diagnosis of type 2 diabetes in 208 premenopausal women with polycystic ovary syndrome. Fertil Steril. 2011;96(5): $1275-1280$.

166. Brohall G, Schmidt C, Behre CJ, Hulthe J, Wikstrand J, Fagerberg B. Association between impaired glucose tolerance and carotid atherosclerosis: a study in 64-year-old women and a meta-analysis. Nutr Metab Cardiovasc Dis. 2009;19(5):327-333.

167. Rosenzweig JL, Ferrannini E, Grundy SM, et al. Primary prevention of cardiovascular disease and type 2 diabetes in patients at metabolic risk: an endocrine society clinical practice guideline. JClin Endocrinol Metab. 2008;93(10):3671-3689.
168. Mosca L. Guidelines for prevention of cardiovascular disease in women: a summary of recommendations. Prev Cardiol. 2007;10 Suppl 4: $19-25$.

169. Timmermans A, Opmeer BC, Khan KS, et al. Endometrial thickness measurement for detecting endometrial cancer in women with postmenopausal bleeding: a systematic review and meta-analysis. Obstet Gynecol. 2010;116(1):160-167.

170. Koss LG, Schreiber K, Oberlander SG, Moussouris HF, Lesser M. Detection of endometrial carcinoma and hyperplasia in asymptomatic women. Obstet Gynecol. 1984;64(1):1-11.

171. Smith RA, von Eschenbach AC, Wender R, et al; ACS Prostate Cancer Advisory Committee, ACS Colorectal Cancer Advisory Committee, ACS Endometrial Cancer Advisory Committee. American Cancer Society guidelines for the early detection of cancer: update of early detection guidelines for prostate, colorectal, and endometrial cancers. Also: update 2001-testing for early lung cancer detection. CA Cancer J Clin. 2001;51(1):38-75.

172. Moran LJ, Hutchison SK, Norman RJ, Teede HJ. Lifestyle changes in women with polycystic ovary syndrome. Cochrane Database Syst Rev. 2011;2:CD007506.

173. Moran LJ, Pasquali R, Teede HJ, Hoeger KM, Norman RJ. Treatment of obesity in polycystic ovary syndrome: a position statement of the Androgen Excess and Polycystic Ovary Syndrome Society. Fertil Steril. 2009;92(6):1966-1982

174. Knowler WC, Barrett-Connor E, Fowler SE, et al; Diabetes Prevention Program Research Group. Reduction in the incidence of type 2 diabetes with lifestyle intervention or metformin. N Engl J Med. 2002;346(6): 393-403.

175. Orio F, Muscogiuri G, Palomba S. Could the Mediterranean diet be effective in women with polycystic ovary syndrome? A proof of concept. Eur J Clin Nutr. Epub 2015 Apr 1.

176. Domecq JP, Prutsky G, Mullan RJ, et al. Lifestyle modification programs in polycystic ovary syndrome: Systematic review and metaanalysis. J Clin Endocrinol Metab. 2013;98(12):4655-4663.

177. Harrison CL, Lombard CB, Moran LJ, Teede HJ. Exercise therapy in polycystic ovary syndrome: a systematic review. Hum Reprod Update. 2011;17(2):171-183.

178. Escobar-Morreale HF, Botella-Carretero JI, Alvarez-Blasco F, Sancho J, San Millán JL. The polycystic ovary syndrome associated with morbid obesity may resolve after weight loss induced by bariatric surgery. J Clin Endocrinol Metabol. 2005;90(12): 6364-6369.

179. Teede HJ, Misso ML, Deeks AA, et al. Assessment and management of polycystic ovary syndrome: summary of an evidence-based guideline. Med J Aust. 2011;195(6):S65-S112.

180. Velazquez EM, Mendoza S, Hamer T, Sosa F, Glueck CJ. Metformin therapy in polycystic ovary syndrome reduces hyperinsulinemia, insulin resistance, hyperandrogenemia, and systolic blood pressure, while facilitating normal menses and pregnancy. Metabolism. 1994;43(5):647-654.

181. Tsilchorozidou T, Overton C, Conway GS. The pathophysiology of polycystic ovary syndrome. Clin Endocrinol (Oxf). 2004;60(1): $1-17$.

182. Nieuwenhuis-Ruifrok AE, Kuchenbecker WK, Hoek A, Middleton P, Norman RJ. Insulin sensitizing drugs for weight loss in women of reproductive age who are overweight or obese: systematic review and meta-analysis. Hum Reprod Update. 2009;15(1): 57-68.

183. Tang T, Lord JM, Norman RJ, Yasmin E, Balen AH. Insulinsensitising drugs (metformin, rosiglitazone, pioglitazone, D-chiroinositol) for women with polycystic ovary syndrome, oligo amenorrhoea and subfertility. Cochrane Database Syst Rev. 2012;5: CD003053.

184. Costello M, Shrestha B, Eden J, Sjoblom P, Johnson N. Insulinsensitising drugs versus the combined oral contraceptive pill for hirsutism, acne and risk of diabetes, cardiovascular disease, and endometrial cancer in polycystic ovary syndrome. Cochrane Database Syst Rev. 2007;(1):CD005552. 
185. Li XJ, Yu YX, Liu CQ, et al. Metformin vs thiazolidinediones for treatment of clinical, hormonal and metabolic characteristics of polycystic ovary syndrome: a meta-analysis. Clin Endocrinol (Oxf). 2011;74(3): 332-339.

186. Tang TLJ, Norman RJ, Yasmin E, Balen AH. Insulin-sensitising drugs (metformin, rosiglitazone, pioglitazone, D-chiro-inositol) for women with polycystic ovary syndrome, oligo amenorrhoea and subfertility. Cochrane Database Syst Rev. 2010;1:CD003053.

187. Nissen SE, Wolski K. Rosiglitazone revisited: an updated metaanalysis of risk for myocardial infarction and cardiovascular mortality. Arch Int Med. 2010;170(14):1191-1201.
188. Gambineri A, Semple RK, Forlani G, et al. Monogenic polycystic ovary syndrome due to a mutation in the lamin A/C gene is sensitive to thiazolidinediones but not to metformin. Eur J Endocrinol. 2008;159(3): 347-353.

189. Talbott E, Clerici A, Berga SL, et al. Adverse lipid and coronary heart disease risk profiles in young women with polycystic ovary syndrome: results of a case-control study. J Clin Epidemiol. 1998;51(5) 415-422.
International Journal of Women's Health

\section{Publish your work in this journal}

The International Journal of Women's Health is an international, peerreviewed open-access journal publishing original research, reports, editorials, reviews and commentaries on all aspects of women's healthcare including gynecology, obstetrics, and breast cancer. The manuscript management system is completely online and includes

\section{Dovepress}

a very quick and fair peer-review system, which is all easy to use. Visit http://www.dovepress.com/testimonials.php to read real quotes from published authors.

Submit your manuscript here: http://www.dovepress.com/international-journal-of-womens-health-journal 